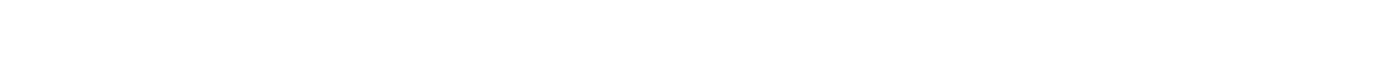

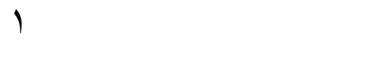

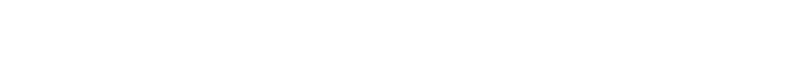

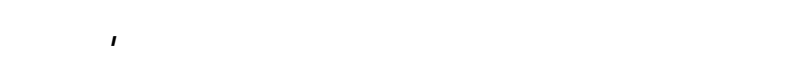

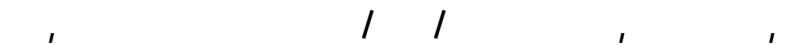

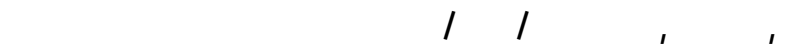

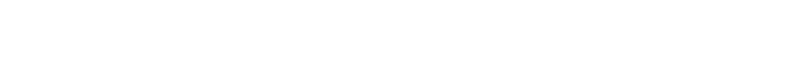

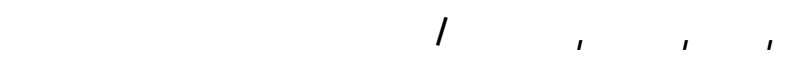
(لالحخار يساوي المفر) حيث يكي بالكاد التلبية الحتيلجلته

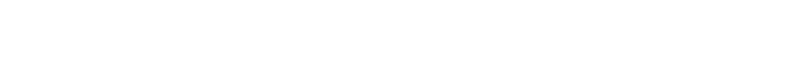

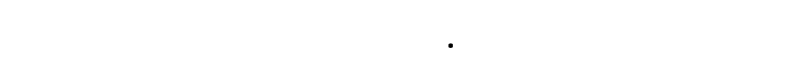

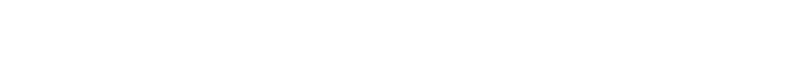

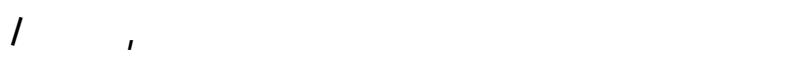

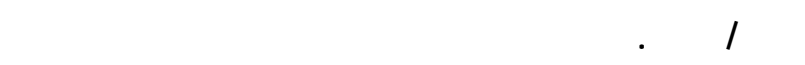

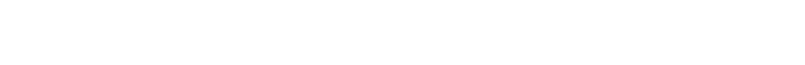
مقارنة بجمع دل المالم المالم.

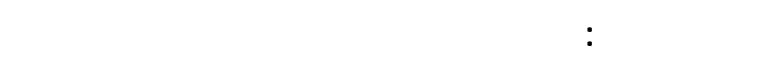

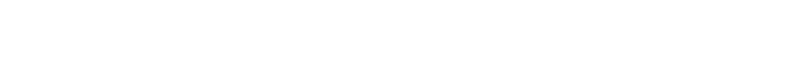

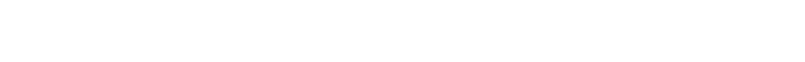
الغنية.

\section{المقدة}

بيتأثر أكثر من خُصْس سـكلن العالم والبالغ عددهم حوالي

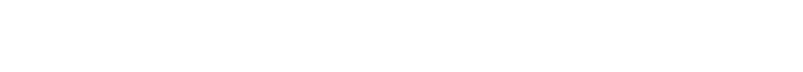

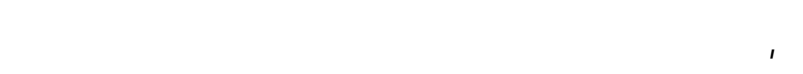

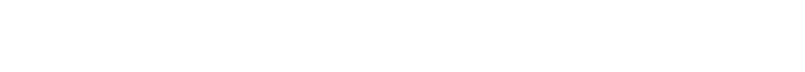

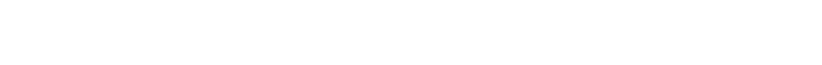
على الغذاء الكافي ونحو r, ا مليار نسمة لا يحصلون على

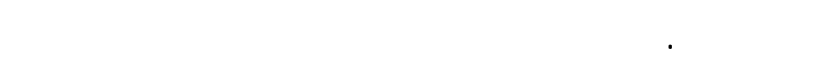

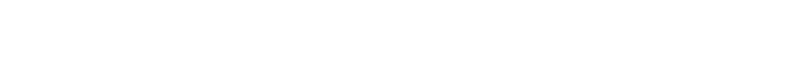

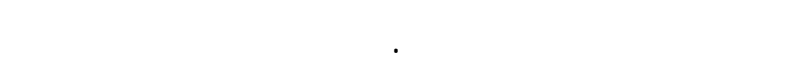

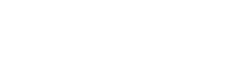

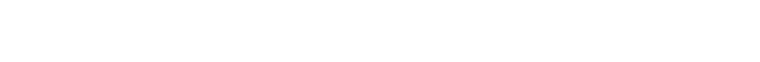
النعاهن الخليه العرب وفارنته بطا النقر الولي، اللعرف

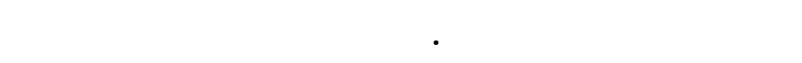

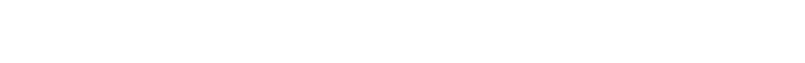

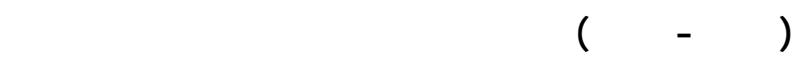

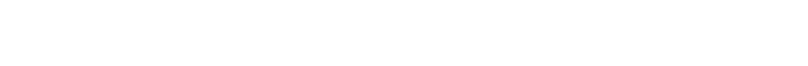

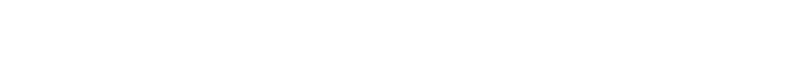

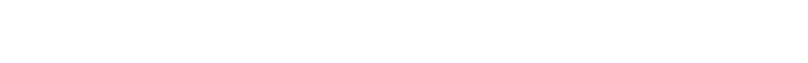

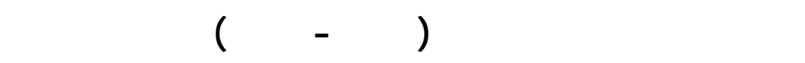
نصيب الفرد الإمارلي من النالج المحل ولالفالق الاستهليكي

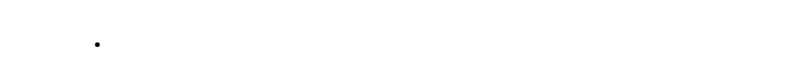

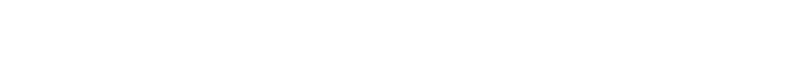

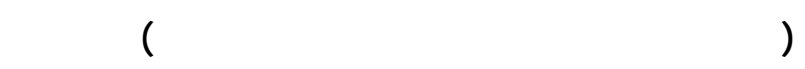

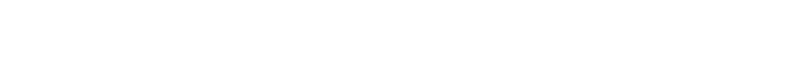

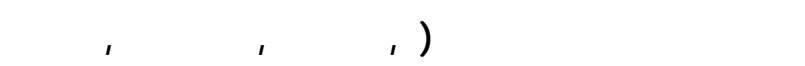

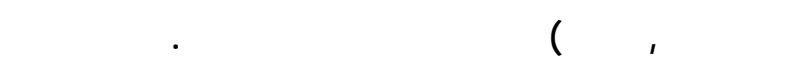

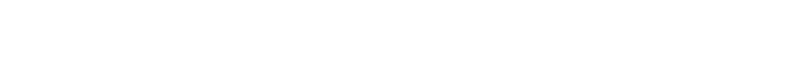

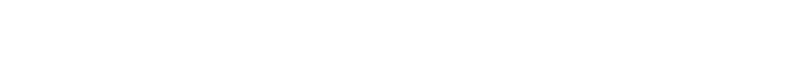

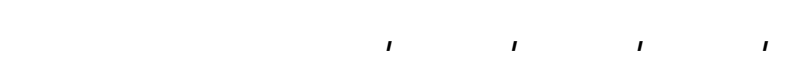
منها عل غن الترتيب.

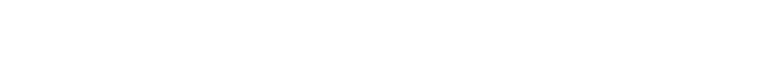

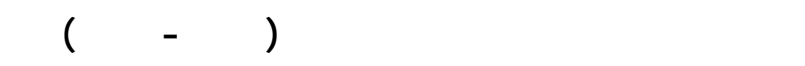

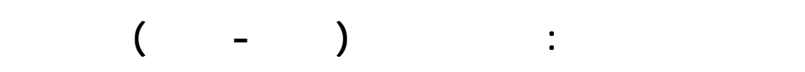

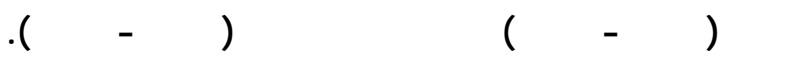

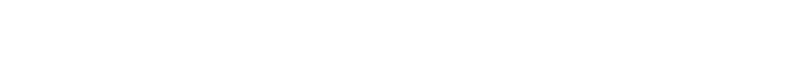

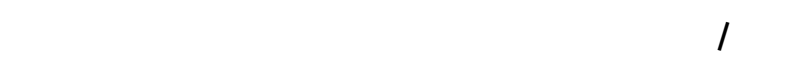

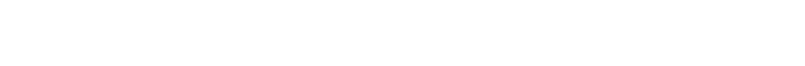
وباشقف الدالة الانخاربة من الدالة الاستهلكية القردرسالفة 
الدول الفقيرة فضل خط الفقر الطلق والدول الغنية فضل

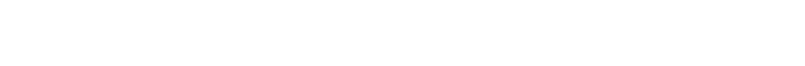

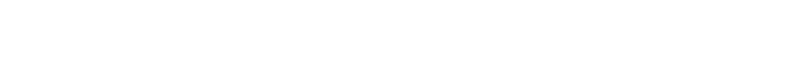

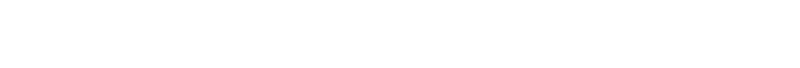

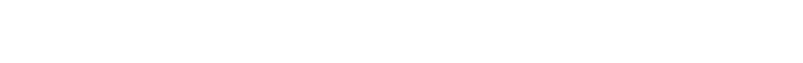

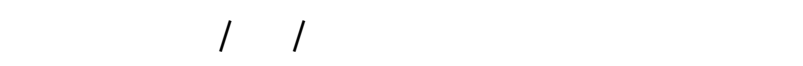
نحو با ا دولار/ يوم/ فرد في الولايت المتحدة الأمريكية.

\section{مشكلة البهث}

يشهد مجلس التعاون الخليجي العربي نمواً القتصاياً

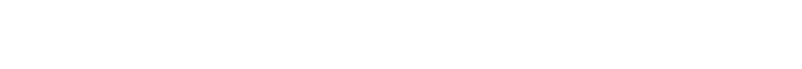

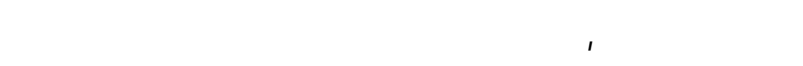

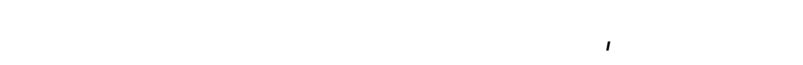

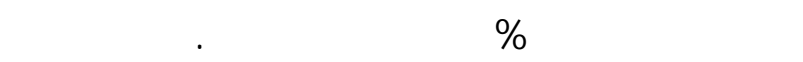

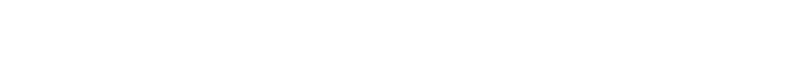

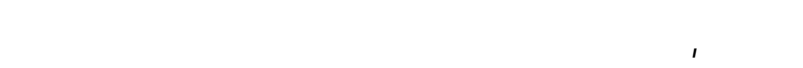

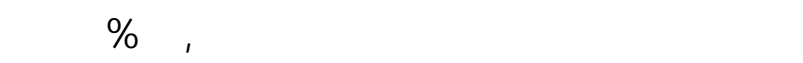

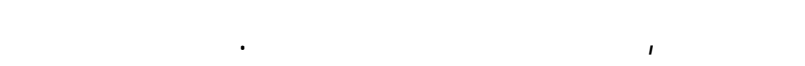

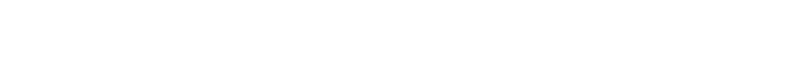
الفرد بدول المجلس والذي يعتمد أسلساً على متوسط الإنفاق اللمستهلاك النهائي للمأسر والبالغ نحو 0\% من من النانج

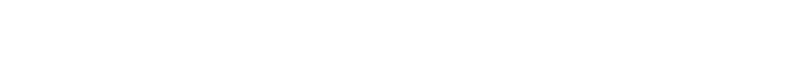

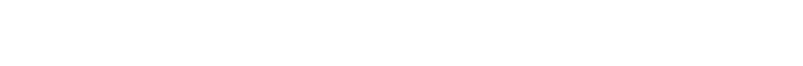

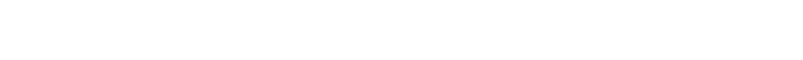

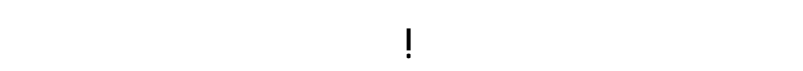

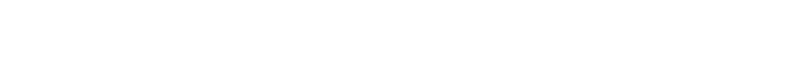

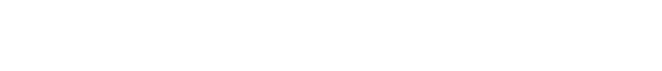

\section{أهدل البه}

يهزف البهث بصفة علمة إلى ققدير خط الفقر المطلق بدول مجلس التعاون الخليجي العربي ومقارنته بنط الفقر

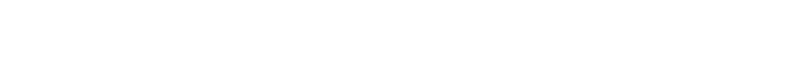
خلال تقهق الأهدف الفرعية التالية:
الدولي للتنمية الزراعية في ققريره حول الفقر فإن نسبة

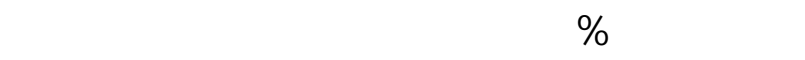
فنس الظروف حق علم ror.r.r. ولن يتمكنوا من الخروج من دائرة الفقر إذا ما لمتمرت معدلات التنمية في الدول النامية على حالها دون تغيير. ومنذ بدلية كقد التسعينات لحتلت قضية الفقر أهمية كبرى على الهيل المستوى العالهي ولجندة العل الدولي النيشهدت عقد عدة مؤتمرات دولية أهنية ناقثت قضايا الفقر.

ويُعد مجطس التعاون لدول الخلبج العربية مظمة

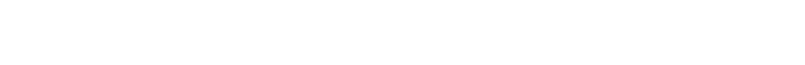
العربي وهي: الإمارات والبحرين وللسعودية وسطلنة عملن

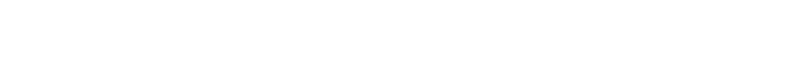

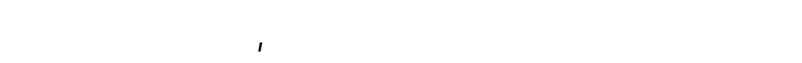

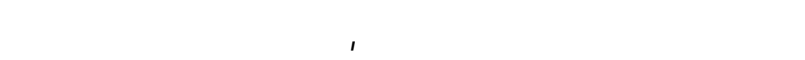

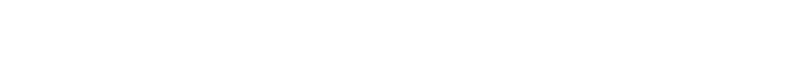

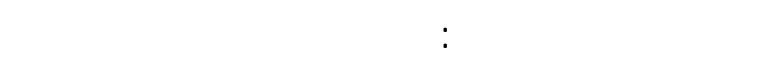

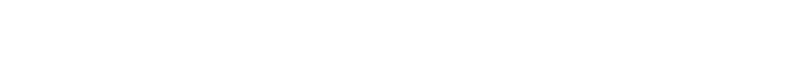
مق توحيد جمبع القيم المالية للمجلس بالدولار الأمريكي. ولا

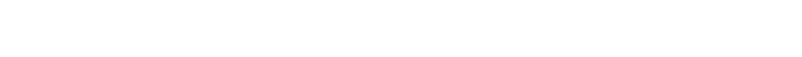

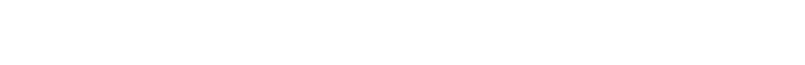
لعل عا. r ع على أنسلس نصيب الفرد من إجمالي النانج المحلي بالدولار الأمريكي وهي: قطر والإمارات والكويت،

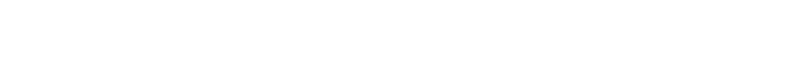

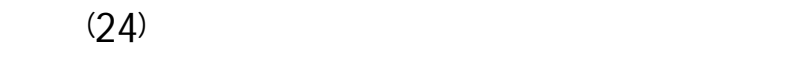
بلغ متوبط نصيب الفرد من إجمالي النانج المحلي في قطر

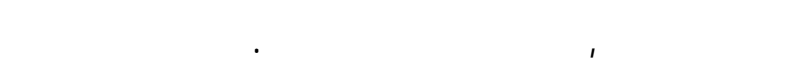

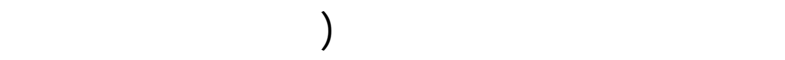

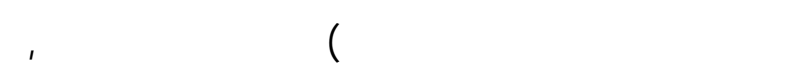

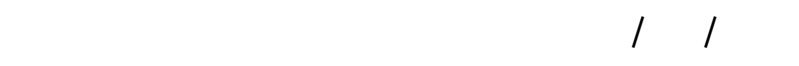
ولكن هذا الطط الدولي لا ينلسب المل الدول حقى بين البلدان

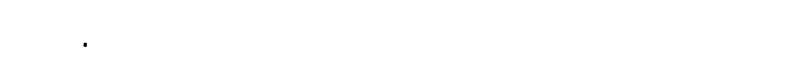
نكر رافاليون -مدير إدارة البحوث في البك الدولي -أن 
العيث يعتبر فقيرًا. وعليه وضعت كل دولة لفنها حداً لطلقت عليه خط الفقر الوطني، وقم تحديه بمبلغ مالي

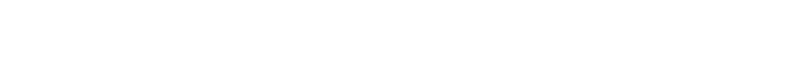

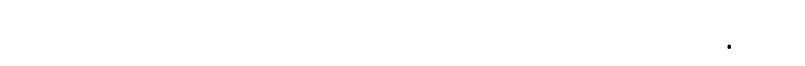

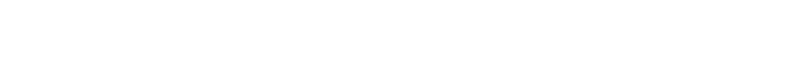

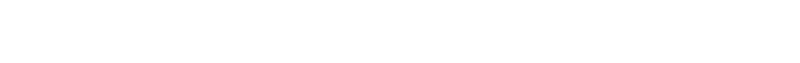
والخملت التعليمية والصحية، وخدملت الصرف الصيح المنلسبة. وقد اعتمدت مظم الدول العربية المفهوم الجديد

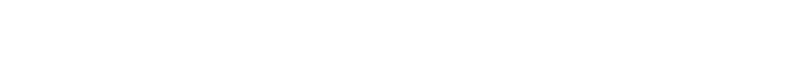

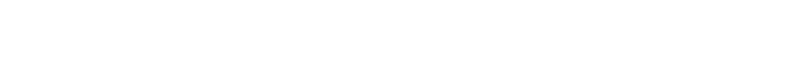
وتشخيصها بشكل دقق. ولستنادَ إلى الأبييت المتوفرة فلِين مفاهيم الققر تشمل الآتي:

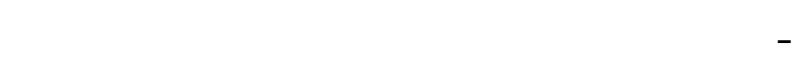

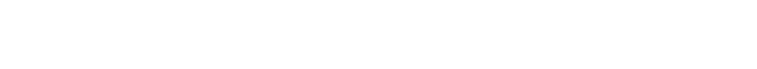

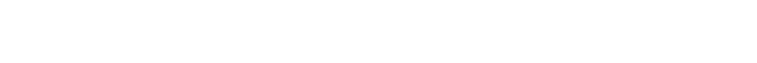

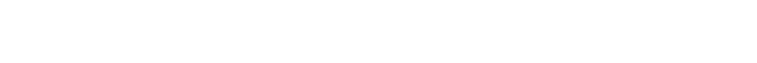
والحرمان من تملك اللسلع المعمرة، وفقدان المقدرة على واله مولجهة الحالات الطارئة كالمرض والطالة والإعالقة والأزمات والكوارث المستدلمة.

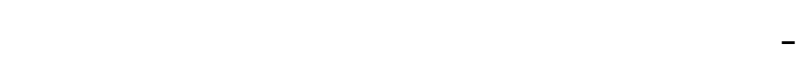

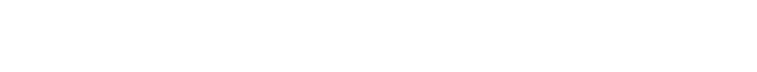

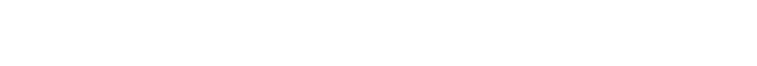
مصادر الميل القية الصالحة للثرب، وعنم توفرمرافق

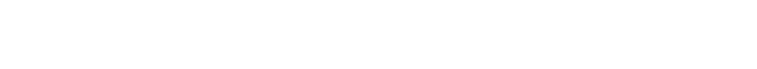
وصعوبة الحصول على عمل أوظظفة ملائمة.

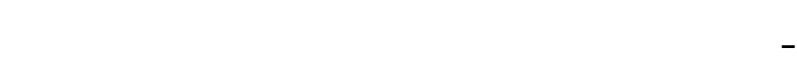

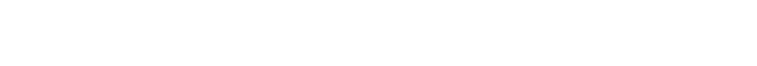

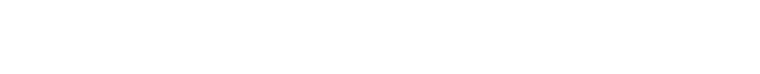
والعناصر الغذائية اللازمة.
- القنير الاحصائي لطورمتونط نصيب الفردمن النانج

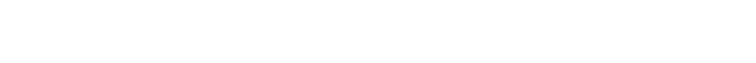

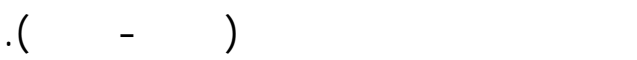
- ققدير دالة المستهلاك الكلي لدول مجلس التعاون

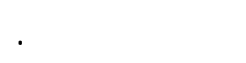
- لمثقاق دالة الانار الكلي لدول مجلس التعاون الخليجي

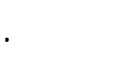
- قدير غط الفتر المطلق على مستوى دول مجلس الفل التعاون الخليجي العربي.

\section{اللمرقة البحثية ومصادر البيلنات}

لتهقيق أهداف الدرلسة مق لمتتخدلم ألسلوبي التحليل

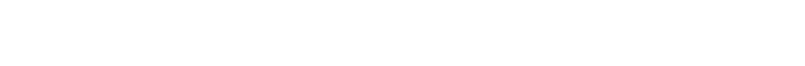

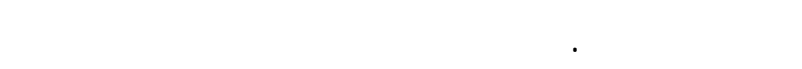

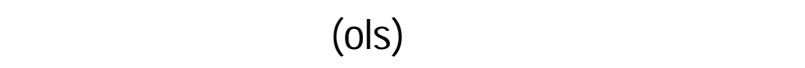
لدول المستهلاك ودول الانخار لدول مجلس التعاون الخليجي العربي، وقدير خط الفقر المطلق لدول مجلس الديل التعاون الخليجي العربي. هذا وقد اعتمدت الدرلمة علي لهيل

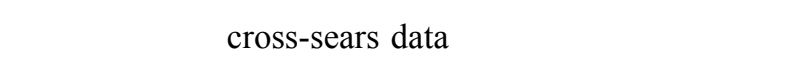

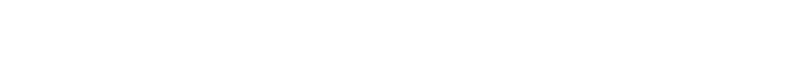

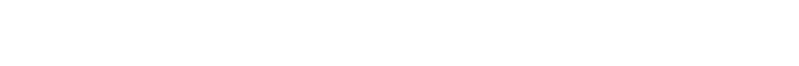
والدرلسلت العلمية ذات الصلة بموضوع البحث.

\section{الإلار الظري للبهث}

يعتبر الفقر ظاهرة إنسانية عميقة الجذور ومتعدة

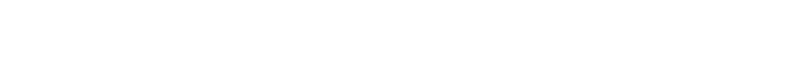
والأزمنة، لذك فقد تباينت آراء الخبراء والبلحثين في تحصيد

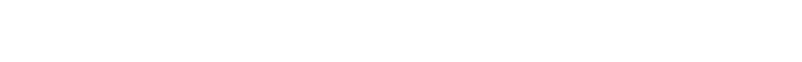

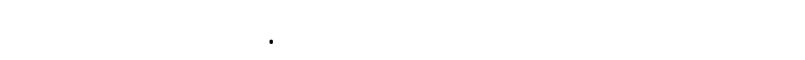

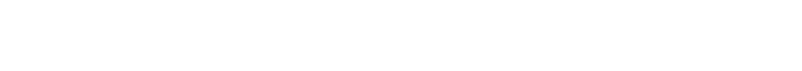

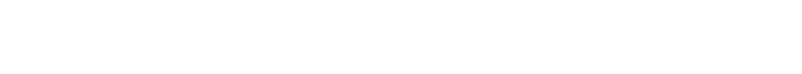

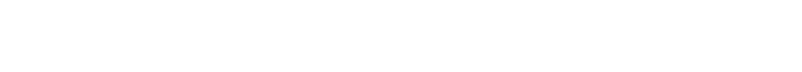

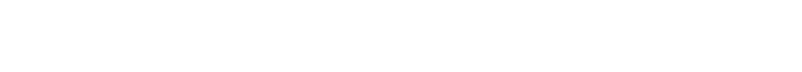


وهذه الرؤية مخالفة لما يؤككه مظم من كتبوا عن الفقر

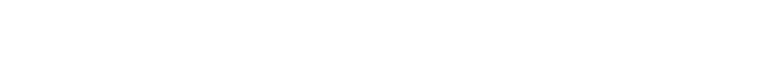

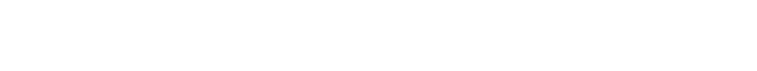

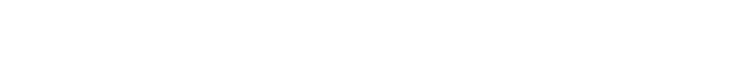
الحياتية التي تعيشها فئت لجتماعية تتسم بالحرملن ف في المجالات المايية والاجتماعية والبيئية.

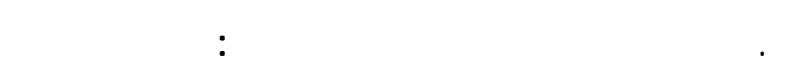

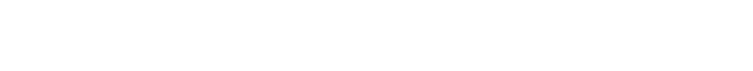
الأتي الفقر: يوصف بالفقر الأفراد والأسر ذات الموارد المجد

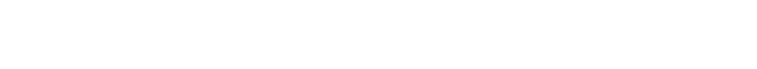

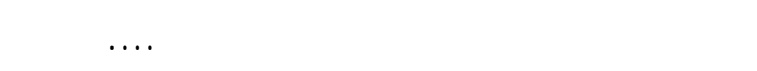

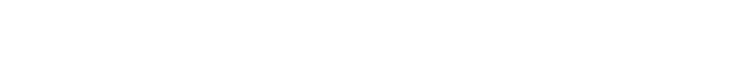

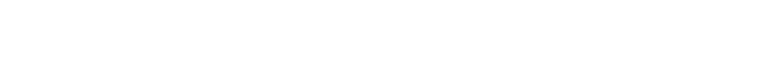
التمتع بحقوق الإنسان جزئيًا أوكلياً. 0.القر البشري: يعني الحرملن من الحية المدية موفورة

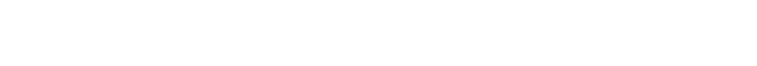
الحصول على ميه للثرب القية والوصول إلى الخملت

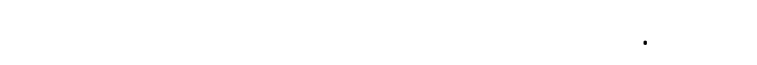

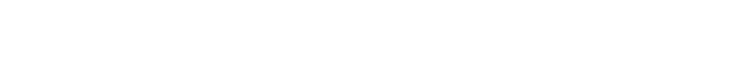
يقم نشرهسنوياً عن بررنلمج الأمم المتح ـة الإنم الئي.

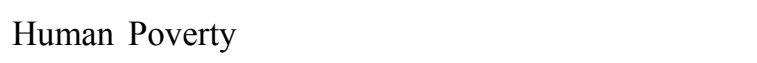
Index كمقيلس تأثيري للاحتيلجلت البثرية. 7.القر الرفي: ارتبط مفهوم الفقر عبر التاريخ بالمنلطق

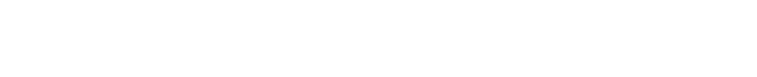

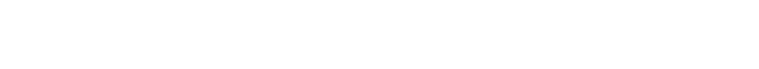

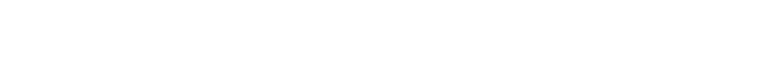

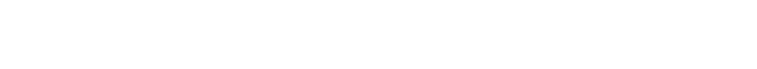
صغيرة. v.الفقر الحضري: ينتثر الفقر الح ضضري بـ بنس كلن الأحياء الصغيرة في المن والت مي تتمي -ز بالازنح لم لم والأحول الصحية السيئة والميه الملوثة، ويسكن فق ـراء المئ
- الحرمان غير المادي كلفقار الإنسن للعبش بحرية

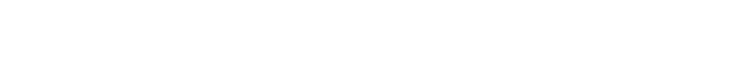

للسيلسية والمدنية.

مستوبت الفقر

ا .الققر الملق Absolute Poverty: يتميز هذا المستوى من الفقر بدرجة عالية من الثبت زمانياً ومكانياً لأنه

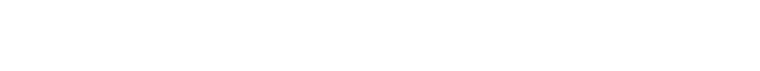

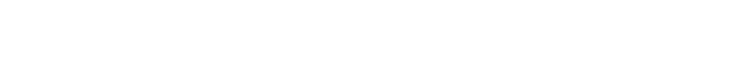
فإن خط الفقر المطلق يساوي لجمالي تكلفة سلة اللسلع الطلوبة لسد الاحتيلجك الإستهلكية الأسلسية وبمعنى

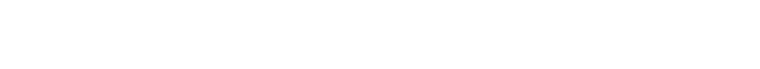
الفسيولوجي، فللثخص يُعد فقيرًا في أية مرحلة زمنية عندما لا تكون لديه القدرة الكفية للوصول إله فئه الموارد

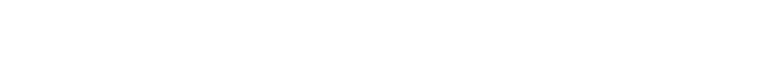

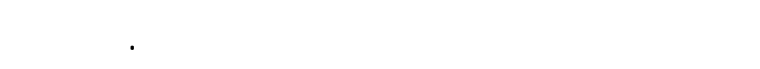

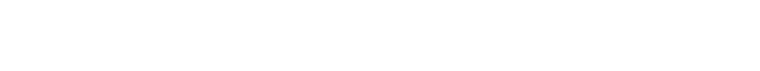

$$
\text { وقت لآخر في البلد فنه. }
$$

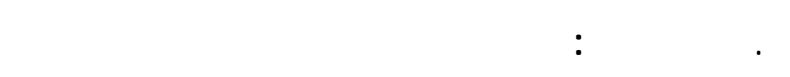

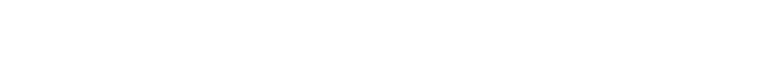

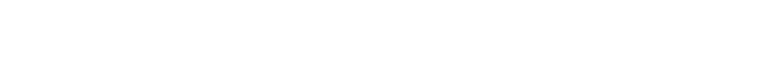

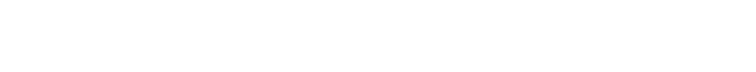
الحية إلا لفترة قصيرة، ولو تحنف خط الفقر المدقع دون

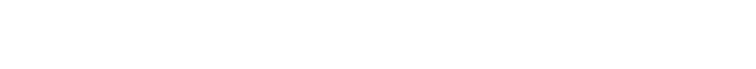
الوار ادمن pauperism

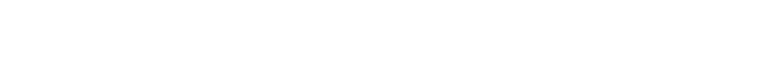

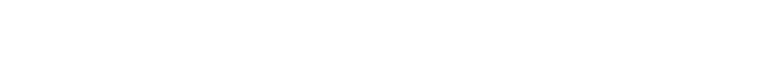
خارجية. ץ. الفقر الجتماعي Social Poverty: ويخرج هذا المعن ق عن المفهوم المادي لأنواع الفقر للسابقة حيث يقصد بـ هـ

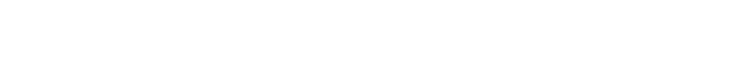

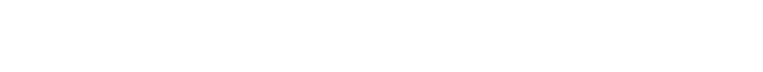


ومريض ومههش ومستبعد لا يعسيش ل سنوات طويل ة وفيقر إله خدمل ضرورية كالصحة ووسالل الإتصل والميه الملمونة وغيرها. ع. .مع قطور قيلسلت الفقر والتعمق في لُّ ـره المفاهيمي ـة

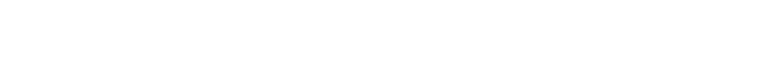

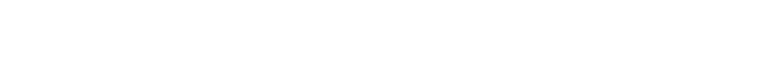

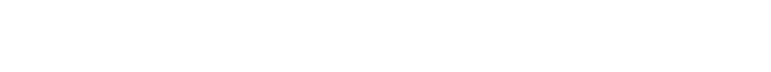

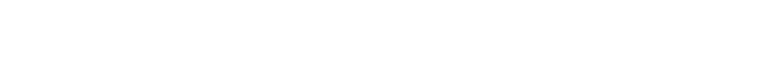

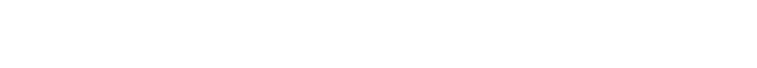

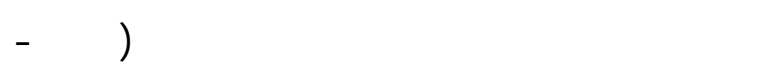

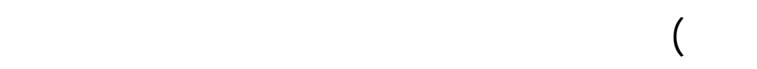

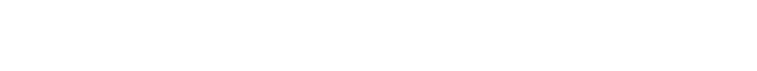
للإحلة باظاواهر على نحوسليم. إن التركيز على الدخل وعلى مستوى المعيشة يعني

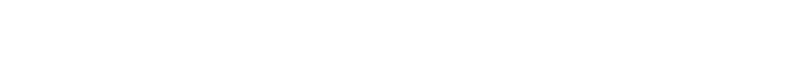
الوطن العربي هو لكثر من مجرد حرملن مادي فهو الإن المعني

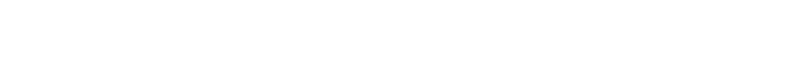
القدرة والتمكين.

طرقفيلس الهقر هنك نلاثطرق رئيسية الفيلس الفقر يمكن الجمالها فيما يالي: أولاً: لستخدلم مؤشر الهقر الستهلي هو الأكثر شيوعًَ ولستخدامًَ في قيلس وتحسيد الفقر وهنالك خمسطرق لتحديد خط الفقر الاستهلاكي: اطرقة الالقة الغذائية: ويقصد بها الحد الأنف من

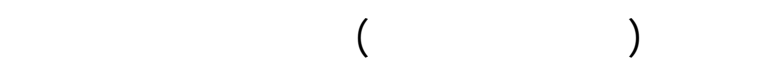
العمليت الحيوية الضرورية لنشط الإنسلن والتي قدرّتها

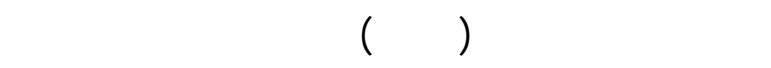
اليوم الولحد.
الحضر عادةً في أحياء أقيمت عشوائيًا حول المدن وغير مصرح بللسكن فيها مما يعرضهر بلستمرار إلى الإخلاء

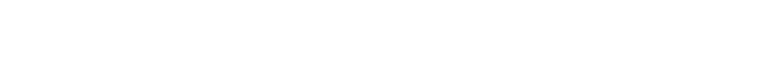

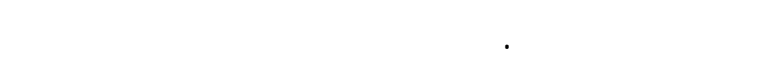

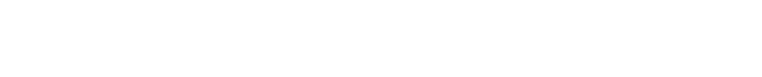
من موجلت الجفل والتصحر أو لشعورهم بعدم الأملن

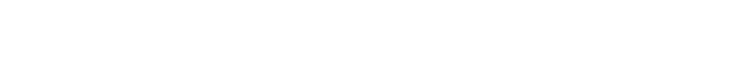

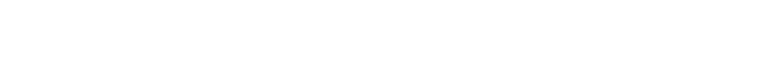

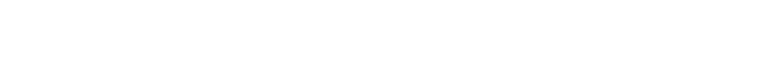
المعيشية.

\section{المؤشرات الخاصة جيطس النقر}

قل التحدث عن طرق قيلس الفقر لابد من لستعراض

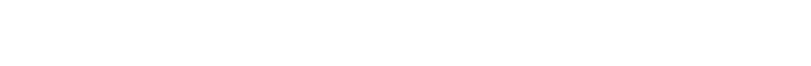

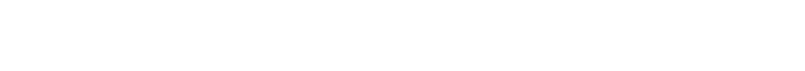
طرق القيلس لاحقاً.... ومن تنك الملاقظلت ما يلي:

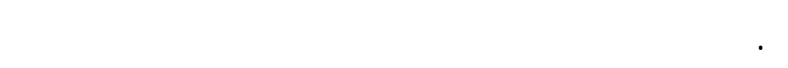

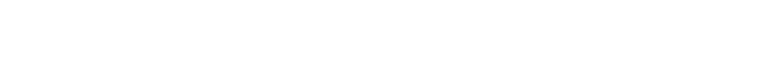

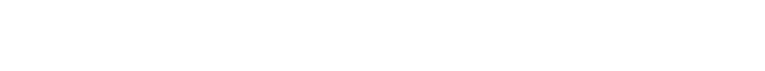

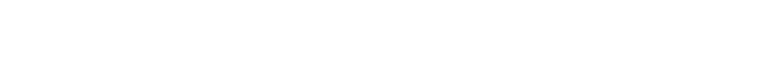
الإجتماعي بدلاًمن الجنس بمعنله البيولوجي. r. لتم الظور في تعريف الفقر بتحول واضح من الكيفية

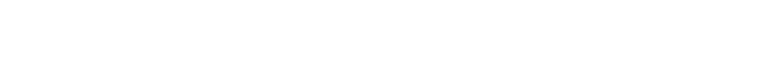

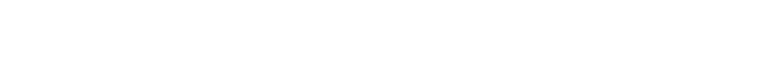

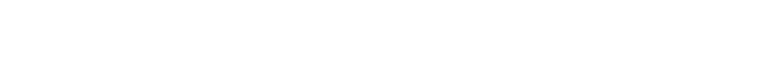

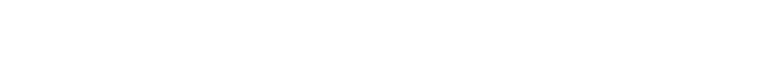
متعدةة. بّ.من الظورات الحاصلة في مفهوم الفق ـر هـ مي ات ساع

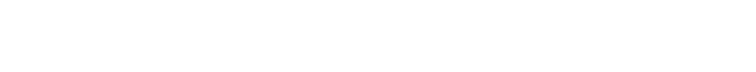

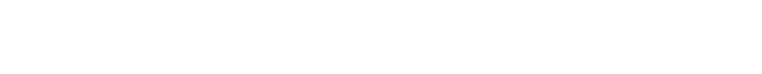
لمعيار وحيد هو الدخل فقد أصح الفقر تعبيرً عن حالة

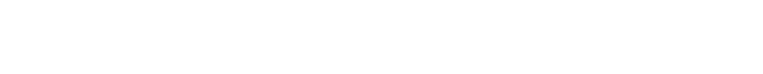


جالب لخر فإن مستوى الرفه للفرد يرتبط إرتبطاً وثيقاً

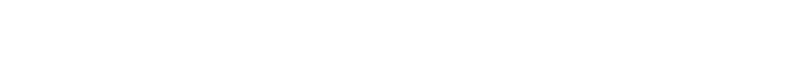

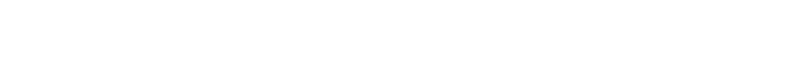
العلمة والمساهمة في النشطلت الاجتماعية وللسيلسية التي

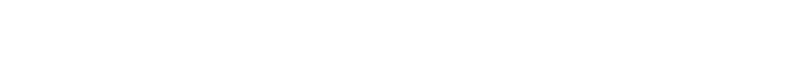
بسبب تلفي مستوى النخول. ثالثاً:مؤشر التنمية البشرية

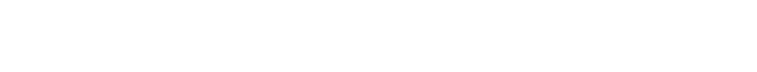

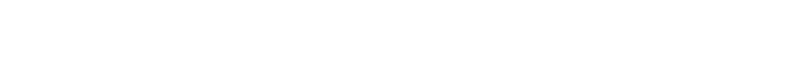
يتميز بكونه يسط الضوء على الأوضاع التنموية البشرية

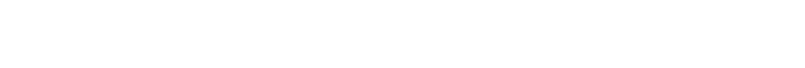

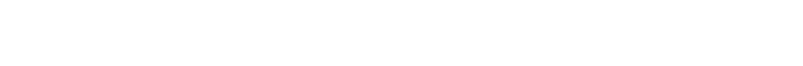
ذك البلد... ويتضمن مؤثر التنمية البشرية عناصر ألسلسية

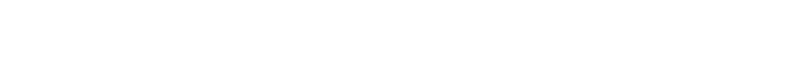
الترجيهي لمعرفة القراءة والكتابة للبالغن ونسب الداسين

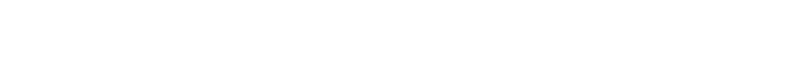
النانج المحلي الإجمالي مقوماً بالدولار الأمريكي وفة التعال التعال

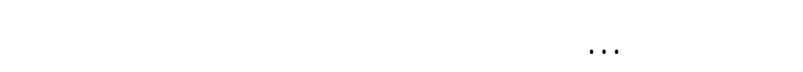

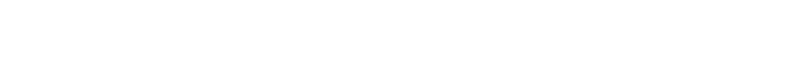
المفثية في ذلك المجتمع منها ما يلي:

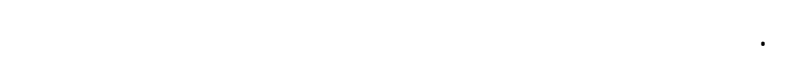
لحتمالية الوفة قبلسن الأربعين. r.حالة الأمية الأبجدية والمعرفية والحرمان من وسالز الإتصل.

ب..الحرمان من المستوى المعيشي اللاقق ويقلس بمتوبط

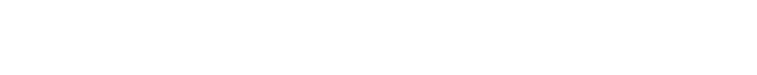

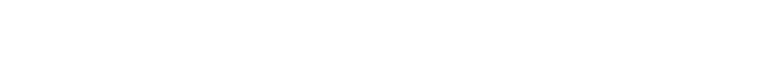
النب المئوية للمعاقين وناقصي الوزن في الألفل. rطرقة تكالف الاحتيلجا الغذائية: وتعني تقدير التكالف اللازمة لشراء وجبة غذائية متوازنة زهية التكالف موصى بها من جهات علمية متخصصة علاوة على الحتيلجلت والمطلبت الأخرى ذات التكلفة المنخفضة. باطريقة حصة الأغنية: وتعتمد هذه الطريقة على نقدير التكلفة الدنيا لسلة غذائية تلبي لحتيلجلت الفرد من الطاقة الغذائية الدنيا. عطرقة خط القر الدولي: وتستخم هذه في أغراض اضن الغنا. المقارنت الدولية، وبموجبها فإن الفرد الذي لا يمكنه الحصول على حزمة لمستهلكية شاملة قبيمة دولار أمريكي ولحد في اليوم (مقوماً بالقوة اللثرائية الثابتة لعلم

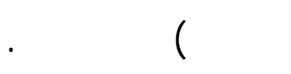

مطرقة اللستهلك النسب: يقم بموجبها تحديد خط الفقر كنسبة مئوية من متوبط المستهلاك الوطني.

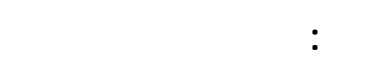
يُعد الفرد فقيرأ مطلقاً إذا لم تمكنه قدراته المالية من المن الحصول على مكونات الحد الأنف للرفه وبتعبير أدق يعتبر

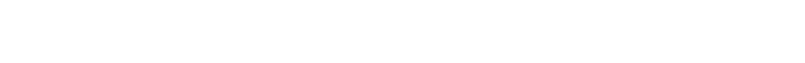

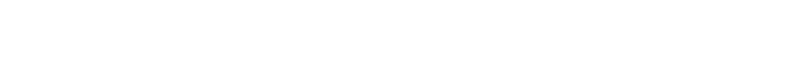
وخدمل الصحة والتعليم ووسائل المشاركة الاجماعية...

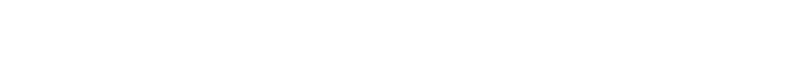

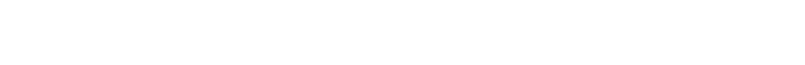

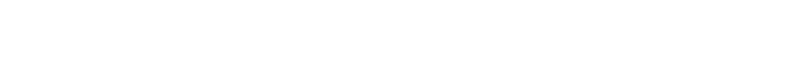

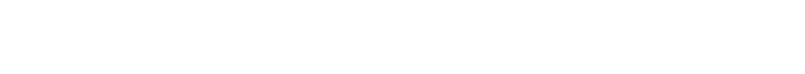

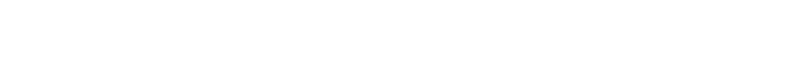

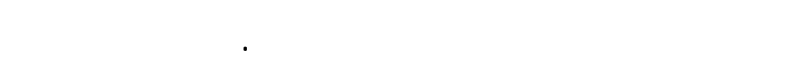

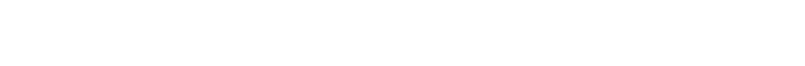

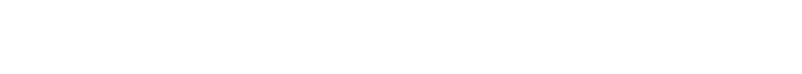
وهو يختلف من فرد لآخر ومن مجتمع لمجتمع كما يختلف وفقاً لتباين المستويات القافية والتكنولوجية اللسائة.... ومن لن 


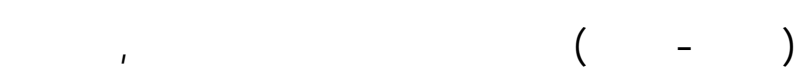

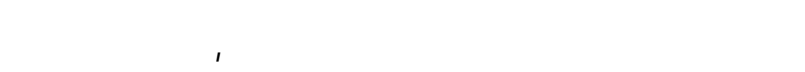
علم عا ·.r. وقد لخذ الناتج المحلي الفردي بالبحرين خلال

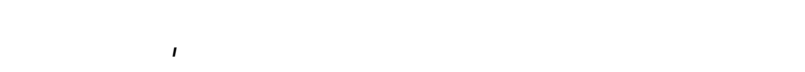

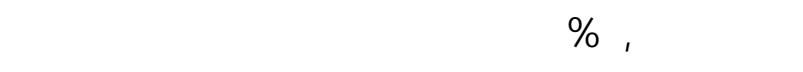

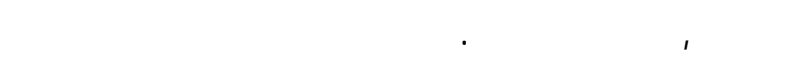

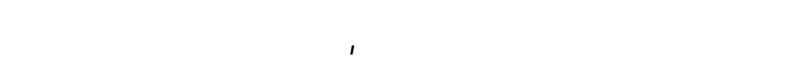
१,، · أي أن 19 \% من التغيرات في متوسط نصيب الفرد البحريني من الناتج المحلي يرجع إلى العوالمل التي يثرحها

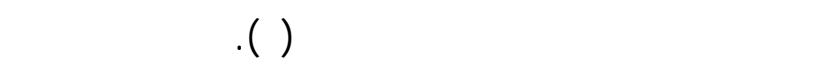

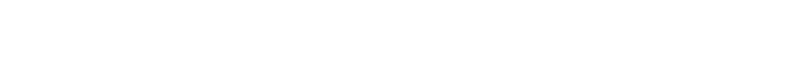

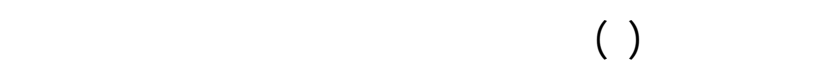

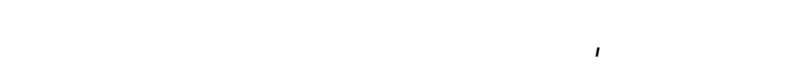

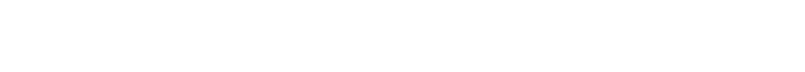

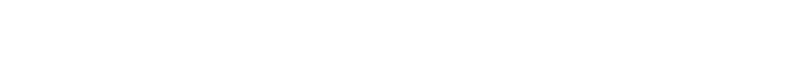

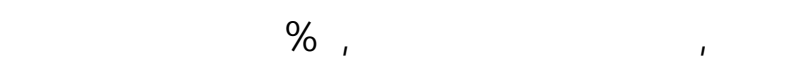

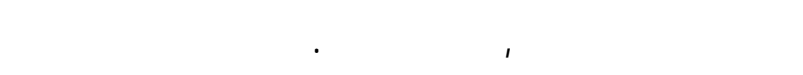

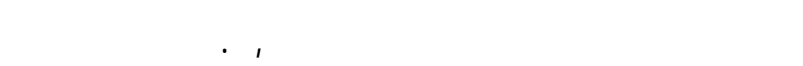
التحنيد 9, · أي أن · 9\% من التغير في الافقاق الإستهاكي لفرد البحريني يرجع إلى العوالمل التي يشرحها متغير

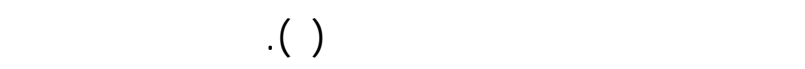
الاستهالكي الفرد بدولة البحرين خلال فترة الدرلسة، التضح مونح

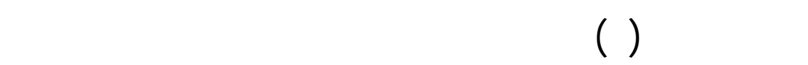

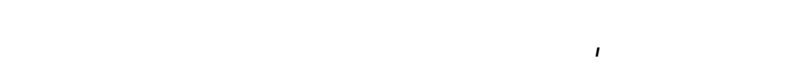

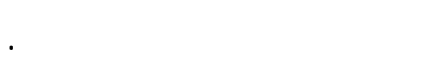

وقد لخذ الإفلق الإستهالكي للفرد البحريني خلل فترة

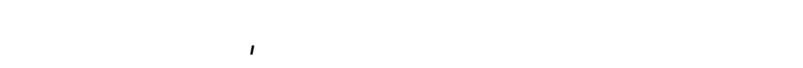

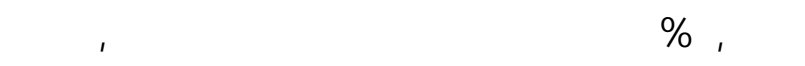

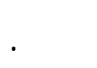

\section{النتائج ومنالثتها}

أولاً: القصير الحصائي لقلور متوطا نصيب الفرد من

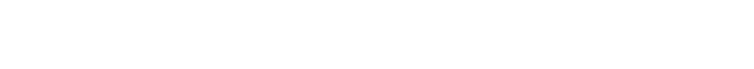

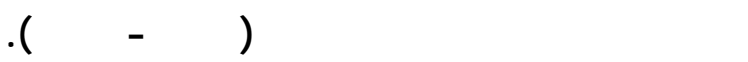
ا ـ الإمارت العربية المتحة:

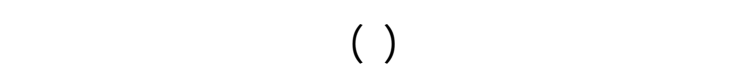
الفرد الإماراتي من الناتج المحلي يتزايد بشكل طفي من

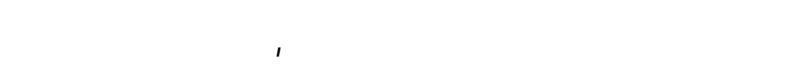

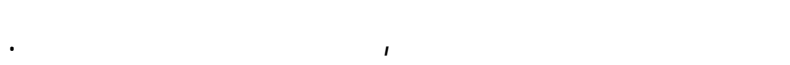
وقد لخذمتوسط نصيب الفردمن الناتج المحلي التجاهاً علماً

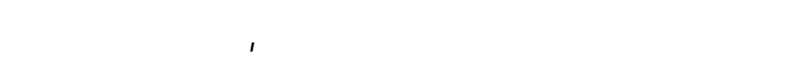

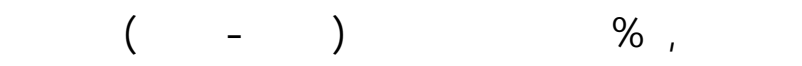

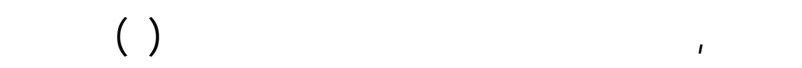
لم تثبت معنوية هذه الزياة عند أي من مستويلت المعنوية الإحصائية المألوفة. الأمر الذي يشير إله لسقةرار متونط

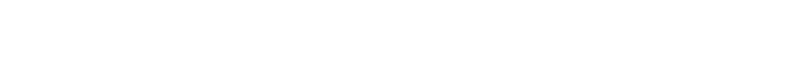

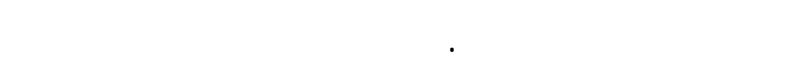

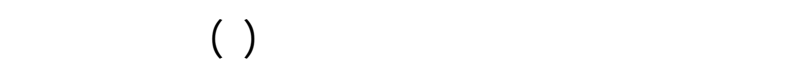

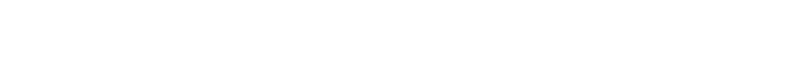

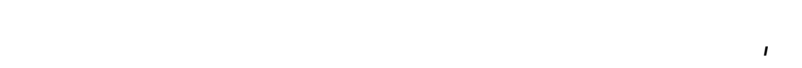

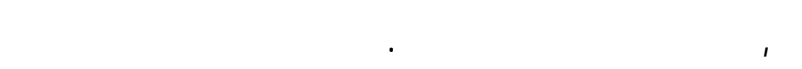

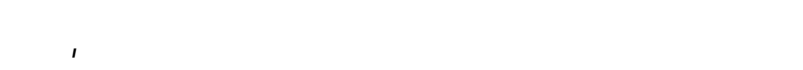
دولار تمل نحو سOب, •\% من متوسط الفترة والبالغة نحو

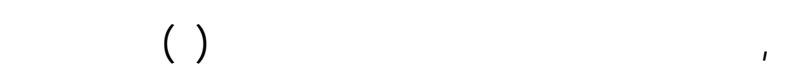

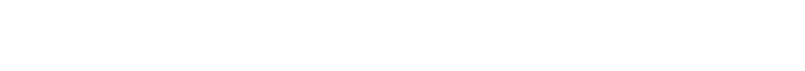
الإحصائية المألوفة. الأمر الذي يشير أيضأ إله لسنقرار

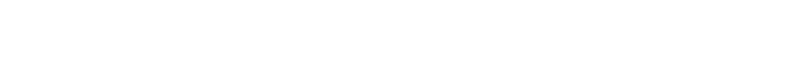
خلال فترة الدرلسة.

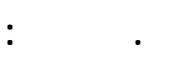

بدرلسة ظطور الناتج المحلي الفردي بدولة البحرين

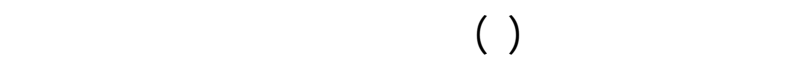




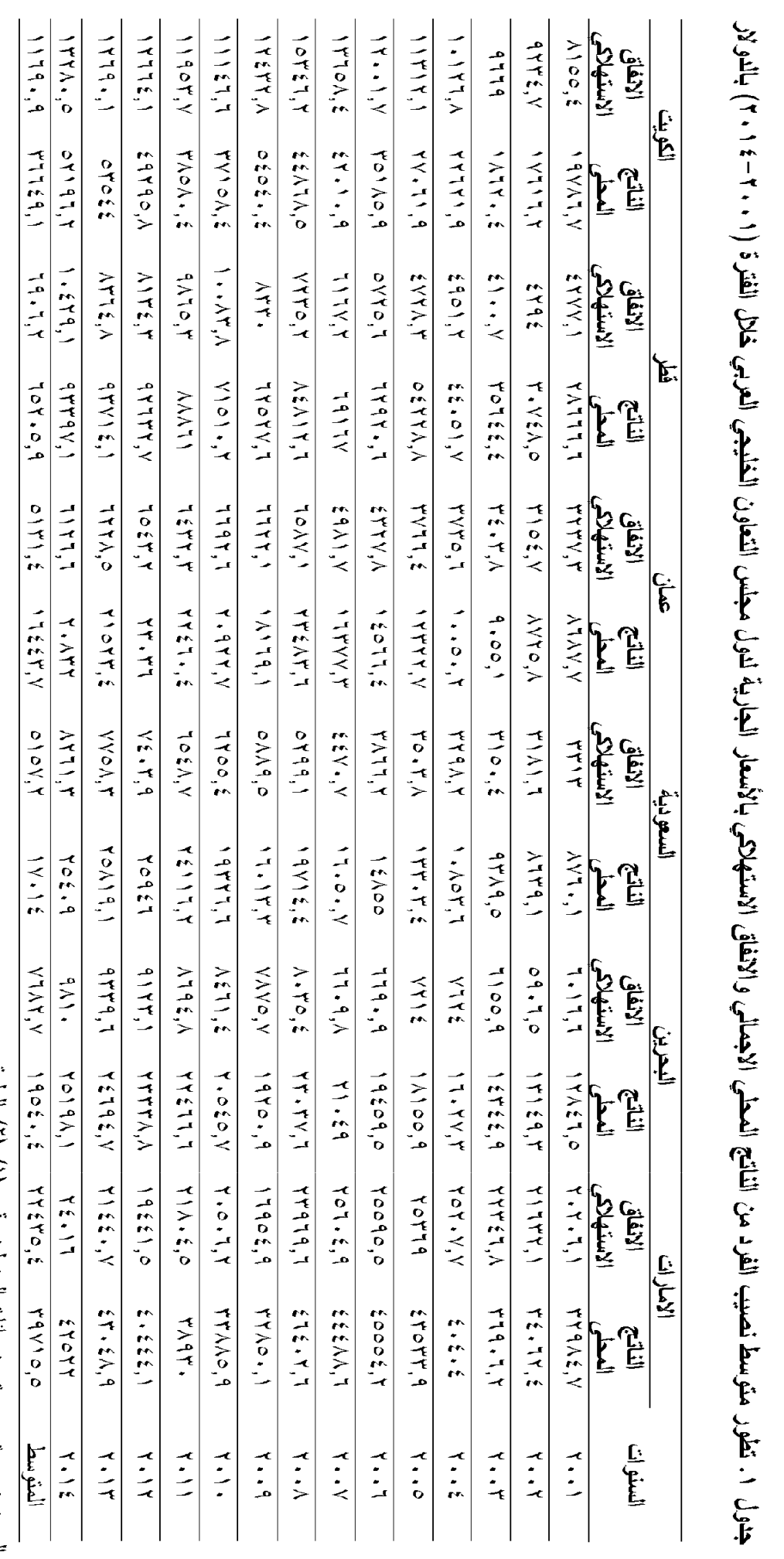




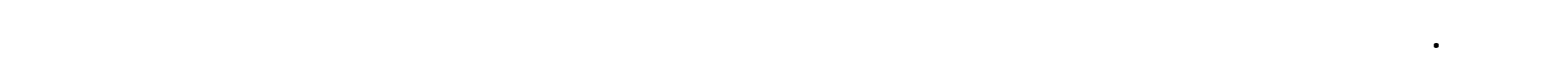

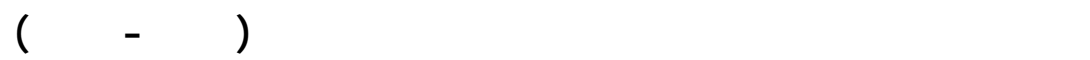

\begin{tabular}{|c|c|c|c|c|c|c|}
\hline معل النمو السنوى \% & $\mathbf{R}^{2}$ & قيمة & $\beta$ & $\alpha$ & المتوسط & المتغير التلع \\
\hline & & & & & & ا. المارات: \\
\hline$\cdot, \Lambda 9$ & $\cdot, 1$ & $(1,1)$ & $\mu 01,0$ & $\mu \nu \cdot \vee १, 0$ & rqV 10,0 & - النانج المحلي الفردي \\
\hline \multirow[t]{2}{*}{$\cdot, \mathrm{OH}-$} & $\cdot, \mu$. & $(\cdot, \mathrm{V}-)$ & $119,7-$ & 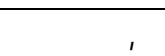 & 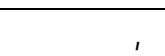 & - الاففاق الاستهالمي \\
\hline & & & & & & r r. البحرين: \\
\hline$\varepsilon, \Lambda$ & $\cdot, 91$ & $*(1 \cdot)$ & $9 \mu \wedge, r$ & $1 \% 0 \cdot \mu, 0$ & $190 \varepsilon \cdot, \varepsilon$ & - النانج المحلي الفردي \\
\hline \multirow[t]{2}{*}{$\mu, \Lambda$} & $\cdot, 9$. & $*(9, \mathrm{~V})$ & rq1,r & O६१८,V & VרAr,V & - الافاق اللستهلامي \\
\hline & & & & & \multicolumn{2}{|c|}{ ب.. المملكة العربية للسعولية: } \\
\hline$\Lambda, \Lambda$ & $\cdot$, १६ & $*(\mid \varepsilon, r)$ & $10 \cdot \varepsilon, \varepsilon$ & $\mathrm{OVH} \cdot, \mathrm{V}$ & $I V \cdot I \varepsilon$ & - النانج المحلي الفردي \\
\hline \multirow[t]{2}{*}{$\Lambda, \varepsilon$} & $\cdot$, १६ & $*(1 \varepsilon, 0)$ & $\varepsilon{ }^{\mu} l, 0$ & $19 r \cdot, \varepsilon$ & $010 V, r$ & - الافقاق الاستهلاكي \\
\hline & & & & & & ع. عمان: \\
\hline$v, 7$ & $\cdot, \Lambda \mu$ & $*(\mathrm{~V}, \mathrm{~V})$ & Irov, & $\mathrm{V} \cdot 1 \cdot, \mathrm{\Lambda}$ & $17 \varepsilon \varepsilon \varepsilon^{\mu}, V$ & - النانج المحلي الفردي \\
\hline \multirow[t]{2}{*}{$7, \cdot$} & $\cdot, \Lambda \cdot$ & $*(7,9)$ & $\mu \| 1, \mu$ & rqvา, & $0|\mu|, \varepsilon$ & - الافلقاق المتهلالمي \\
\hline & & & & & & 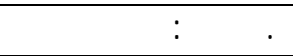 \\
\hline$\Lambda, \mu$ & $\cdot, 9$ & $*(1 \cdot, \Lambda)$ & OEl. & $r \varepsilon \Gamma^{\mu} \cdot, 9$ & $70 r \cdot 0,9$ & - النانج المحلي الفردي \\
\hline \multirow[t]{2}{*}{$\mathrm{V}, \varepsilon$} & $\cdot, \Lambda \varepsilon$ & $*(\Lambda, Y)$ & $0 \cdot V, q$ & $\mu \cdot 97,7$ & $79 \cdot 7, r$ & - الافقلق الاستهلالمي \\
\hline & & & & & & ا 7. الكويت: \\
\hline$V, 9$ & $\cdot, \Lambda$ & $*(\mathrm{~V})$ & $r \wedge 91, \mathrm{~V}$ & Iह११७,ह & $\mu 77 \varepsilon q, 1$ & - النانج المحلي الفردي \\
\hline$r, \Lambda$ & $\cdot, 0$ & $*(\mu, \varepsilon)$ & TYY & $9 \mathrm{qVV} 0,0$ & $1179 \cdot 9$ & - الافاق الاستهلمي \\
\hline
\end{tabular}
المصدر: جمعت ومسبت من بيانت الجدول رقم (1) بالبهث.

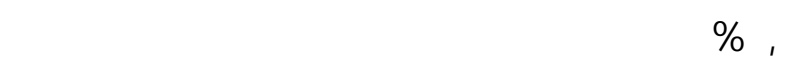

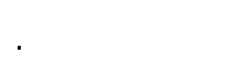

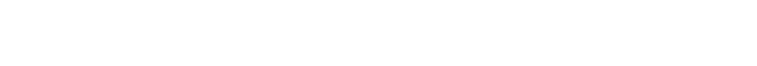

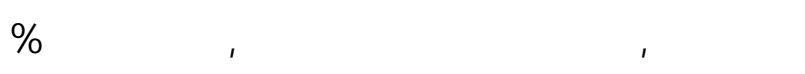

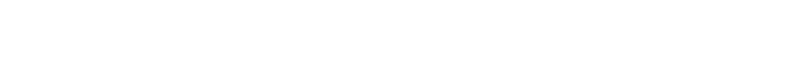

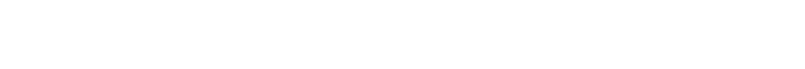

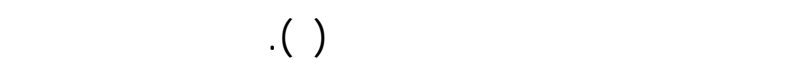

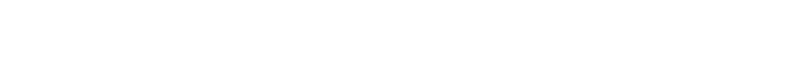

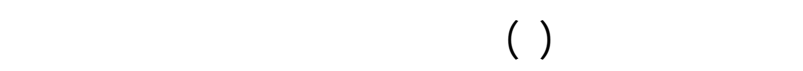

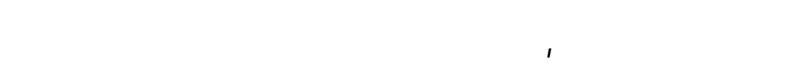

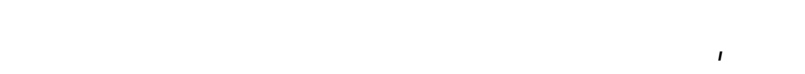

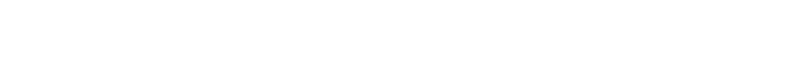

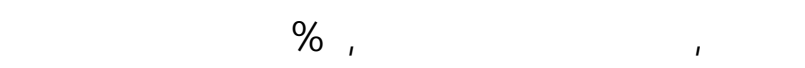

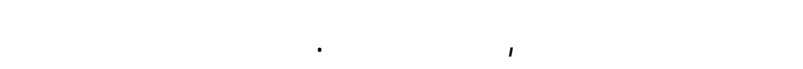

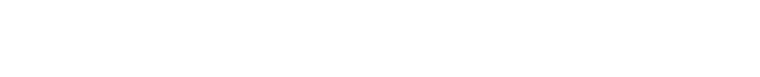

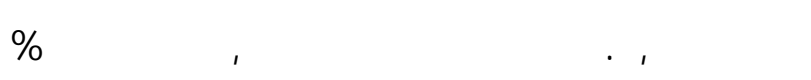

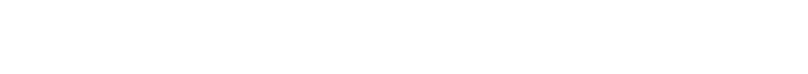

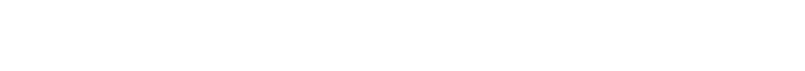

\section{r. الممكة العربية اللسعونية:}

بدرلسة طور متونط نصيب الفرد اللسعودي من النانج

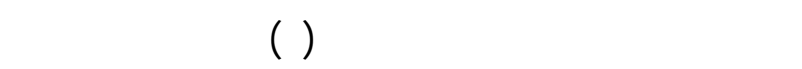

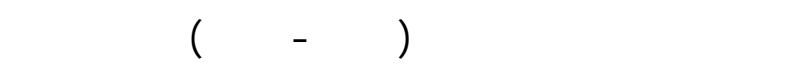

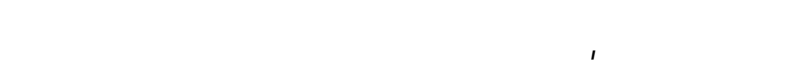

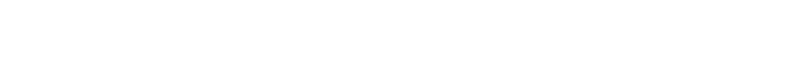

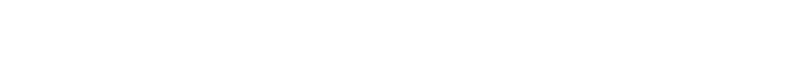

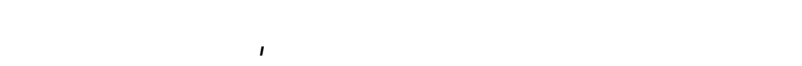




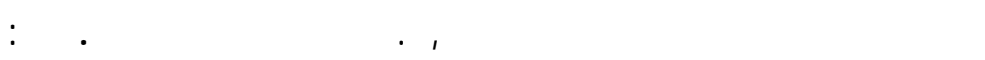
بدرلسة ظوور متوسط نصيب الفرد الثطري من الناتج

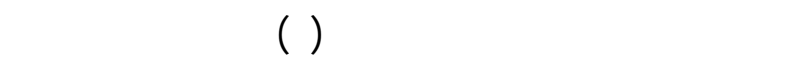

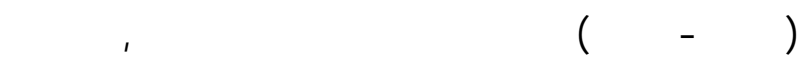

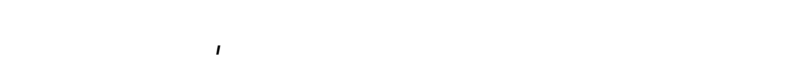

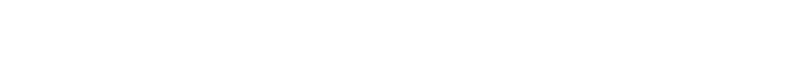

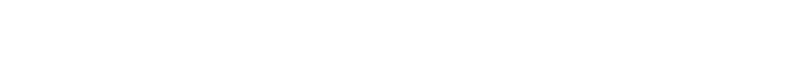

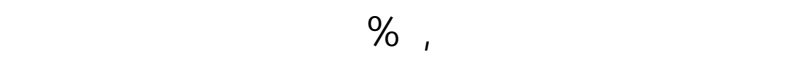

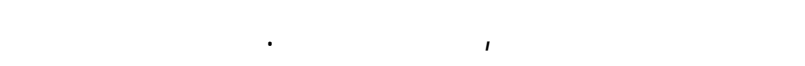

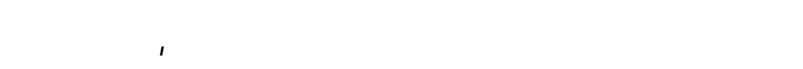

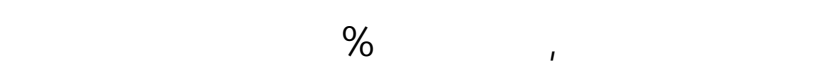

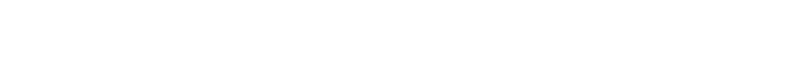

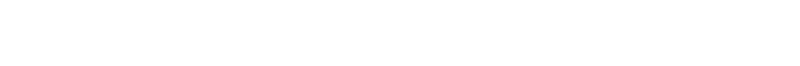

وبالنسة للإفاق المستهلامي للفرد التطري خلل فترة

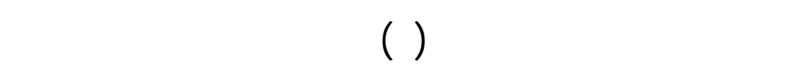

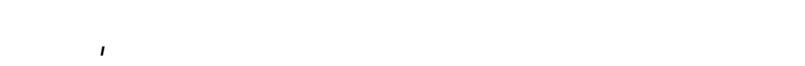

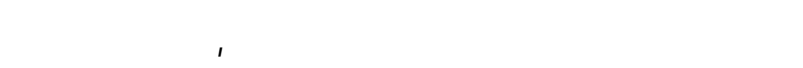

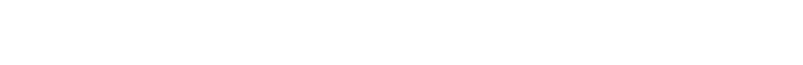

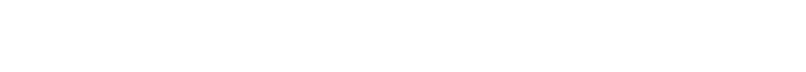

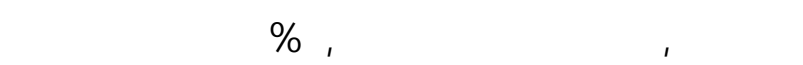

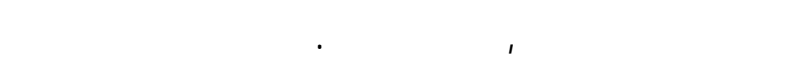

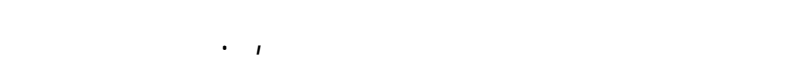

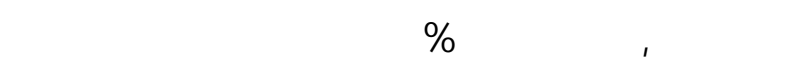

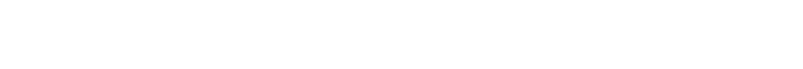

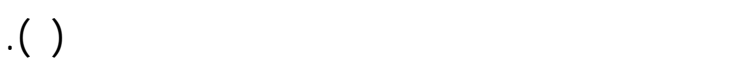

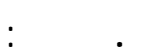
بدرلسة قطور متوسط نصيب الفرد الكويتي من النانج.

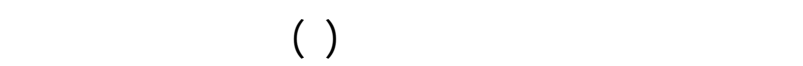

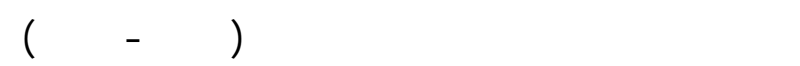

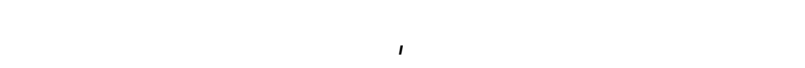

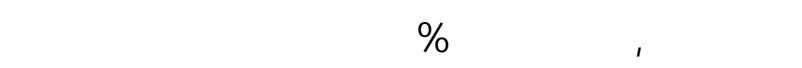

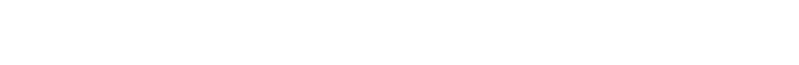

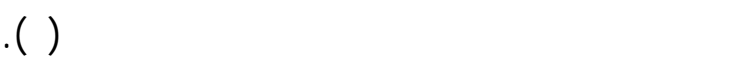

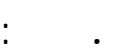
بدرلمة ظطور متوطط نصيب الفرد العماني من النانج

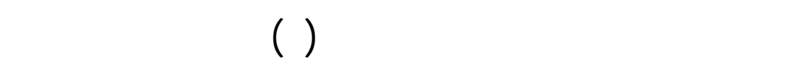

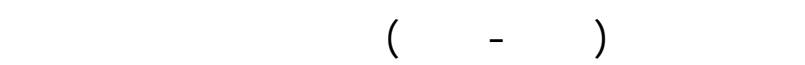

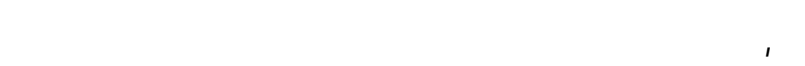

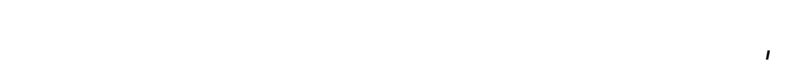

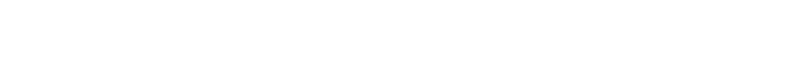

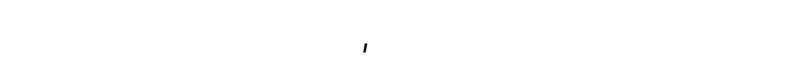

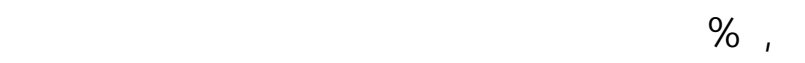

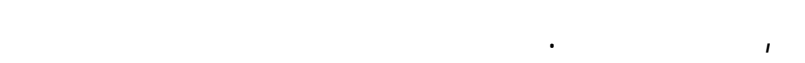

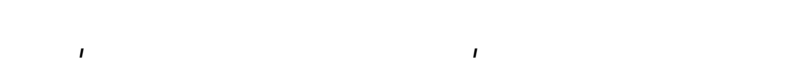

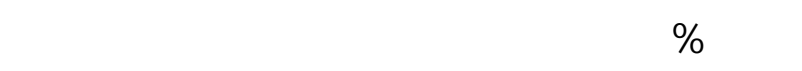
النانج المحلي الاجمالي يرجع إلى العوامل الني يشرحها

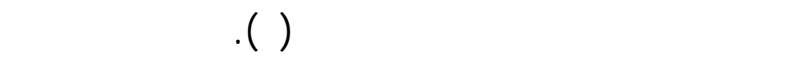

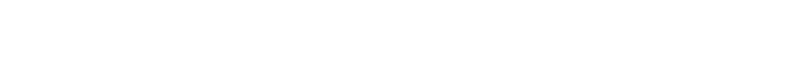

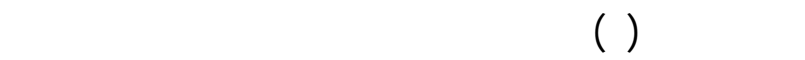

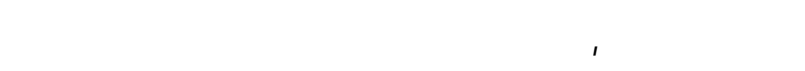

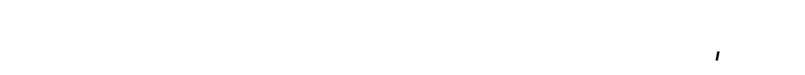

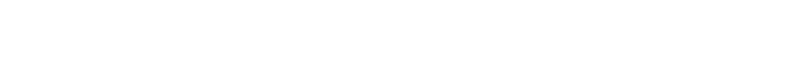

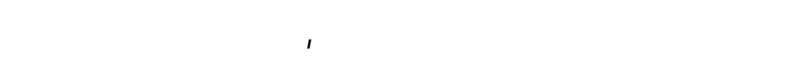

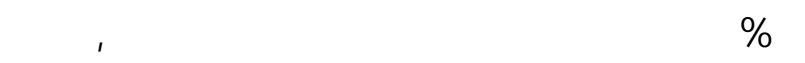

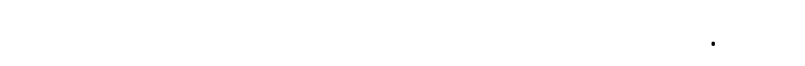

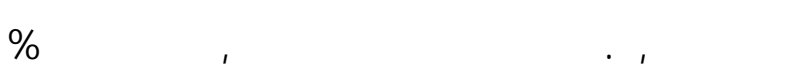

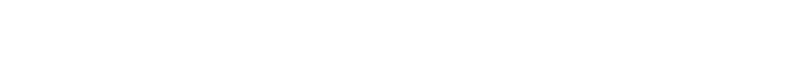

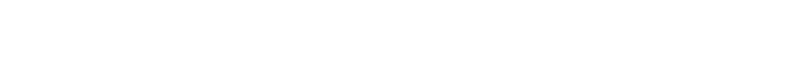
كما هوموضح بالجدول(r). 
وبمعلومية الدالة الاستهلكية المقدرة بلستخدلم الموذج

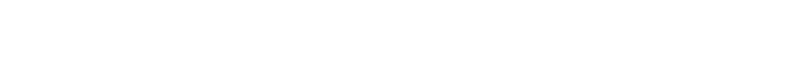
التعالل للنخل بدالة الانخار والذي يمكن تعريفه بأنه التهانية

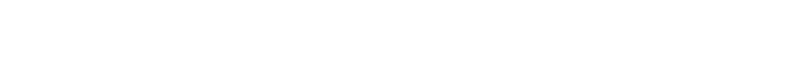
الاستهلاكية الأسلسية للفرد، ويمكن لثنقاق هذا النخل عند النديل مساوة الانخار بالصفر، ومن مُ فإن الفرد الذي لخله دون هذا المستوى يقع تحت خط الفقر المطلق.

\section{فير خط الققر الملاق علل مستوى دل مجطس النعاهن}

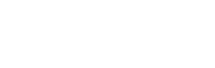

باللستعانة بالنموذج الاحصائي اللساق وبيانت الجدول

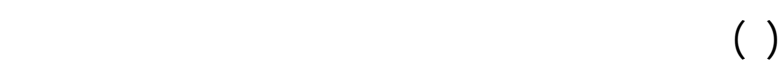

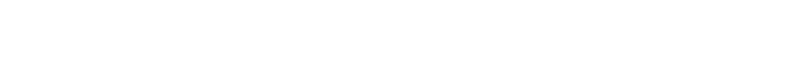

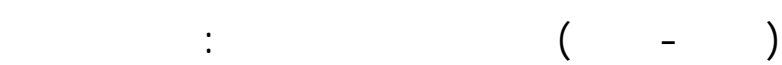

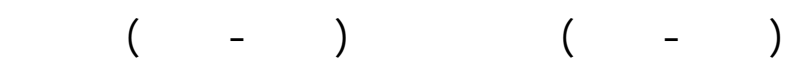

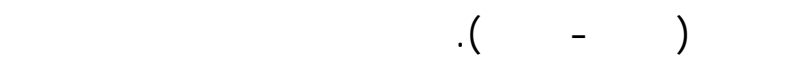

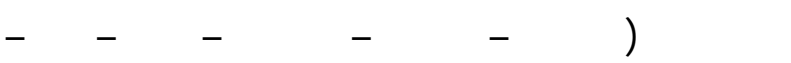
الكويت) بطريقة cross-series data. وبلستخدلم لأسلوب الانحدار البيط وطريقة المربعلت الصغرى العاية(ols)

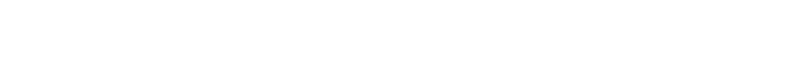

\section{| ـ العترة الأول ( ا . . - -ع - . م):}

بلستعراض نتائج القدير الاحصائي للفترة الأوله

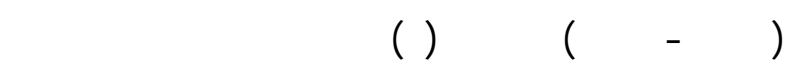

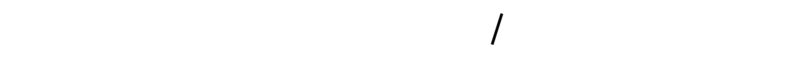

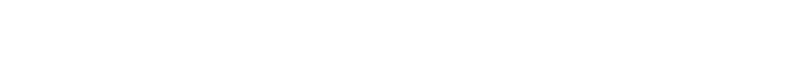

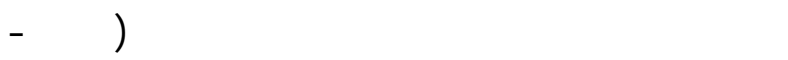

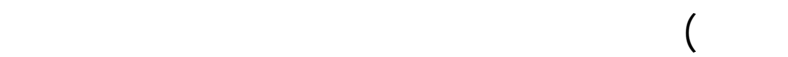

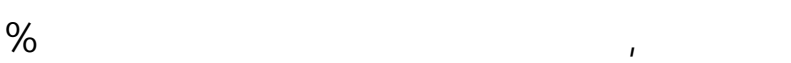
من التغيرات التي ظرأ على قيمة الافلق الإستهاكي معلي للسنوي للفرد بدول المجلس ترجع إلى التغير في الدخل

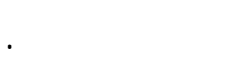

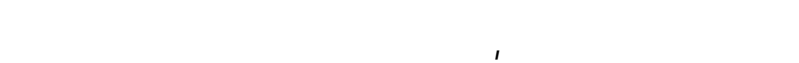
الناتج المحلي الفردي بدولة الكويت خلل فترة الدرلسة

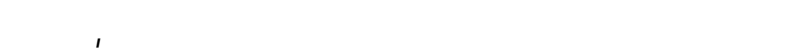
دولار تمل نحو 9, و من متوسط نصيب الفرد من الناتج

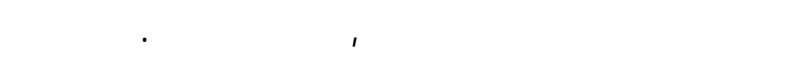

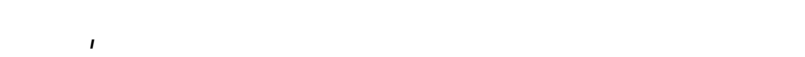

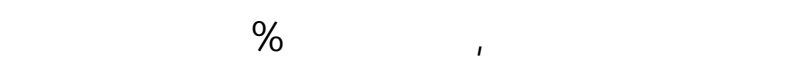
متوسط نصيب الفرد الكويتي من النانتج المحلي يرجع إله

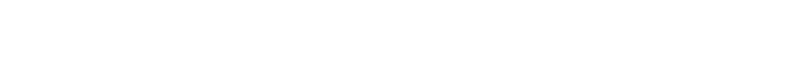

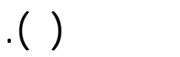
وبالنسبة للإففاق الاستهلاكي للفرد الكويتي خلل فترة

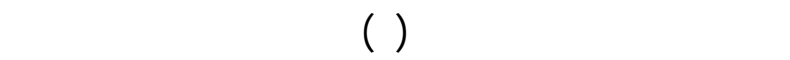

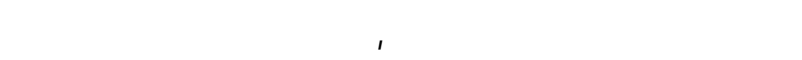

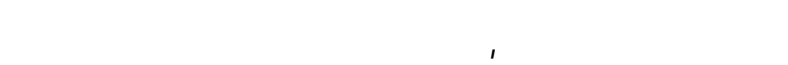
الانفق الإستهلكي للفرد بدولة الكويت خلال فترة الدرلسة

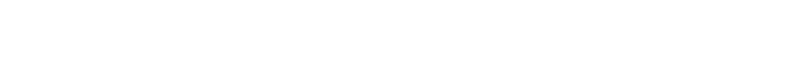

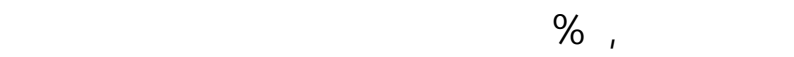
9, ·1199 دولار. وقد شبت معنوية هذه الزياة إحصائياً

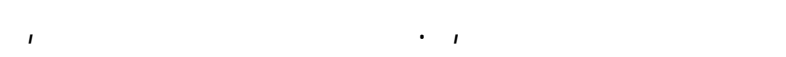

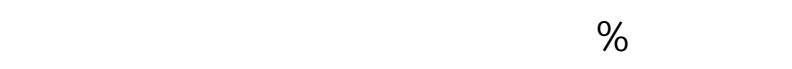
الكويتي يرجع إلى العولمل التي يشرحها متغير الزمن كما

هوموضح بالجدول(r) .

\section{شانياً: القحير الإحصائي للا الفر الملقاق}

اعتمدت الدرلسة في نقديرها لدالة الاستهلاك الكلي

(لجمالي الاستهلاك) للفرد على النموذج الاحصائي التالي: $\mathrm{C}_{\mathrm{ij}}=\alpha+\beta \mathrm{I}_{\mathrm{ij}}+\varepsilon_{i}$

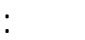

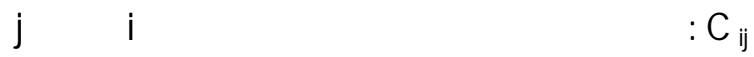

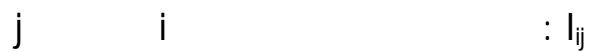
i م، م ثوالبت المعادلة. 


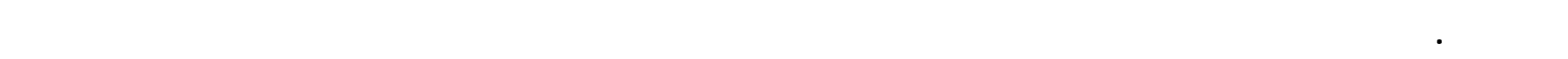

الدرلمة الثلاثة

\begin{tabular}{|c|c|c|c|}
\hline 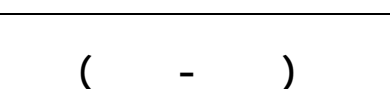 & 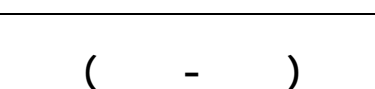 & ( الفترة - الأولى) & الدالة الفقرة \\
\hline $\mathrm{C}_{\mathrm{i}}=9664.2+0.03 \mathrm{I}_{\mathrm{i}}$ & $\begin{array}{c}\mathrm{C}_{\mathrm{i}}=5944.2+0.12 \mathrm{I}_{\mathrm{i}} \\
(3.5)^{*} \\
(2.7)^{*}\end{array}$ & $\begin{array}{l}\mathrm{C}_{\mathrm{i}}=752.9+0.47 \mathrm{I}_{\mathrm{i}} \\
(0.2) \quad(3.9)^{*}\end{array}$ & النموذج المقدر لد \\
\hline $\mathrm{R}^{2}=0.02 \quad \mathrm{~F}=1.4$ & $\mathrm{R}^{2}=0.11 \quad \mathrm{~F}^{*}=7.5$ & $\mathrm{R}^{2}=0.40 \quad \mathrm{~F}^{*}=15$ & \\
\hline $\mathrm{S}_{\mathrm{i}}=-9664.2+0.97 \mathrm{I}_{\mathrm{i}}$ & $\mathrm{S}_{\mathrm{i}}=-5944.2+0.88 \mathrm{I}_{\mathrm{i}}$ & $\mathrm{S}_{\mathrm{i}}=-752.9+0.53 \mathrm{I}_{\mathrm{i}}$ & دالة الاخخارالمستنطة \\
\hline $\begin{aligned}\left.\mathrm{I}_{\mathrm{i}} \mid \mathrm{S}_{\mathrm{i}}=0\right)= & (9664.2 / 0.97= \\
& 9963.1\end{aligned}$ & $\begin{array}{c}\left.\mathrm{I}_{\mathrm{i}} \mid \mathrm{S}_{\mathrm{i}}=0\right)=(5944.2 / 0.88= \\
6754.7\end{array}$ & $\begin{array}{l}\left.\mathrm{I}_{\mathrm{i}} \mid \mathrm{S}_{\mathrm{i}}=0\right)=(752.9 / 0.53= \\
1420.5\end{array}$ & مستوى التعال للحخل \\
\hline $9963.1 / 365=27.3$ & $6754 / 365=18.5$ & $1420.5 / 365=3.9$ & دولار / الئر المقدر الفرد/ \\
\hline
\end{tabular}

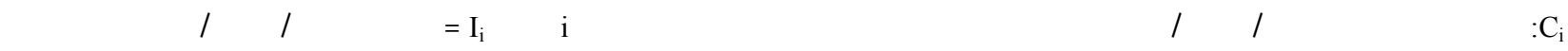

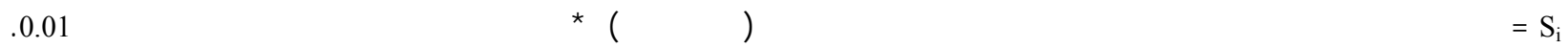

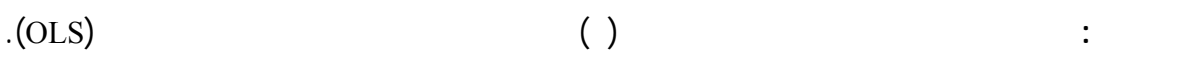

الخليجي العربي ترجع إلى التغيرات في الدخل اللسنوي لهذا الفرد. وعند لمثقاق الدالة الانخارية من الدالة الاستهلكية

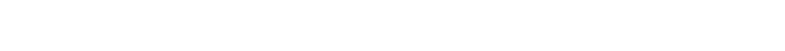

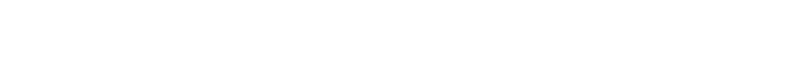

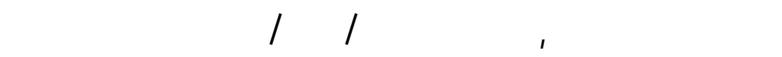

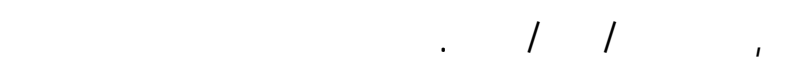

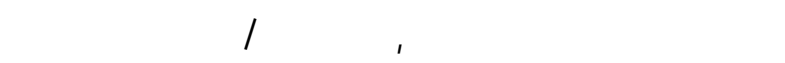
جزء من نخله (الانخار يساوي الصفر) حيث يكفي بالكاد

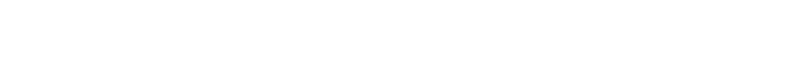
هذا الحد لن يسطبع تلبية لحتيلجاته الاستهلكية.

\section{r.الفترة الثالثة ( • ( · -ع ا · م):}

بلستعراض نتائج القدير الاحصائي الفترة الثالثة

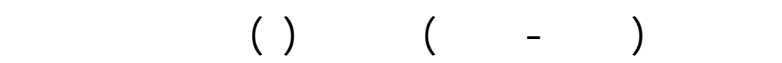

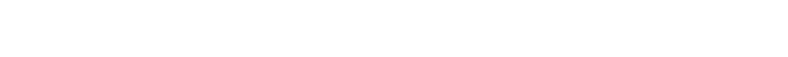
مجلس التعاون الخليجي العربي خلال تلك الفترة إلا أنه لم

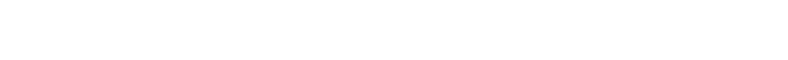
المعنوية الاحصائية المألوفة. وعند الشقاق الدالة الانخارية

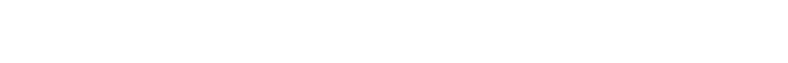

وبلشقاق الدالة الانخارية من الدالة المستهلكية المقدرة

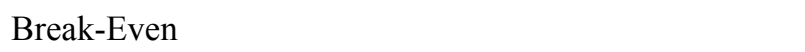
والتضح منه أن خط الفقر المطلق للفرد Level of Income

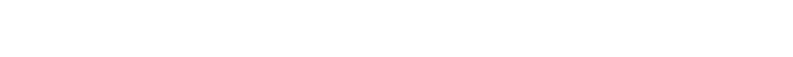

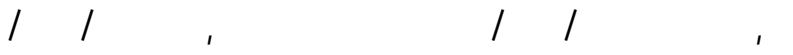
اليوم، وهذا يعني أن الفرد خلال تاكل الفترة والذي نخله

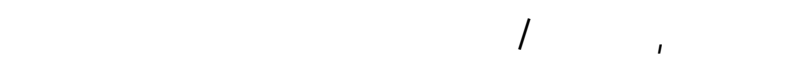
(الآخار يساوي الصفر)حيث يكفي بالكاد لتلبية لحتيلجاته

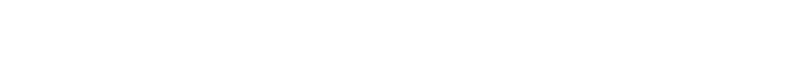
يسطبع تلبية لحتيلجاته المستهلكية.

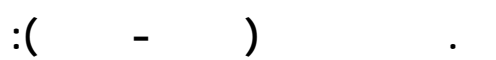

بلستعراض نتائج القدير الاحصائي الفترة الثانية

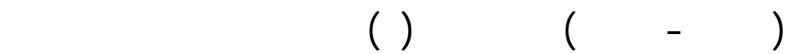

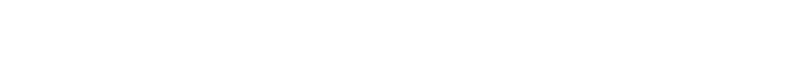
التعاون الخليجي العربي خلل تالك الفترة وقد ثبتت معنوية هذه الزياة لحصائيًا عند مستوى معنوية 0 ., ولثأشارت

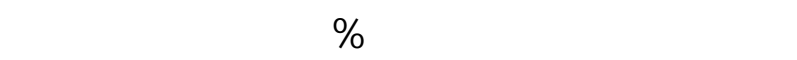
على قيمة الاففق الاستهلاكي للفرد بدول مجلس التعاون 
وأن IV أف طفل يولجهون الجوع القانل يومياً، ويعقد

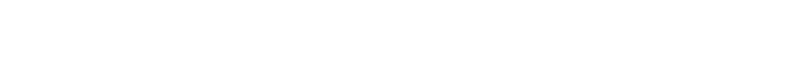
تعود إلى قص التغنية، ولقد حقق عدد من الدول العربية الخدية

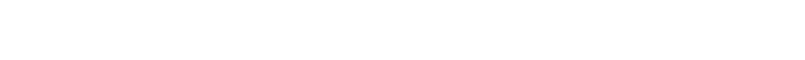
ملحوظاً فيشأن تخفيض نسبة اللسكلن ناقصي التغنية كما

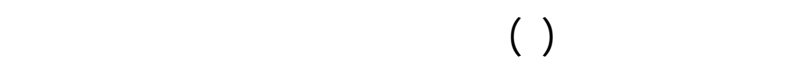
نسبياً في بلدان لخرى من دول الوطن العربي التي تأثرت

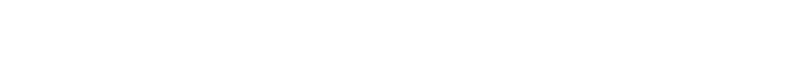

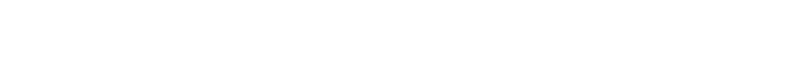
النبة في دول مجلس التعاون بين 0, ار\% في الكويت

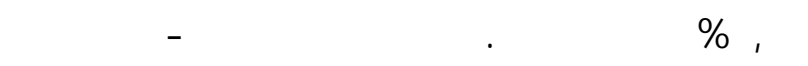

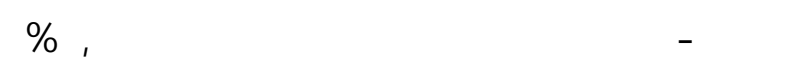

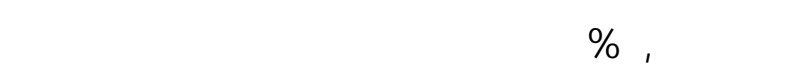

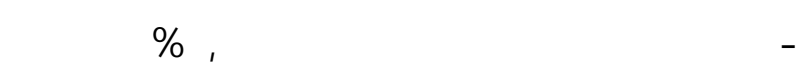

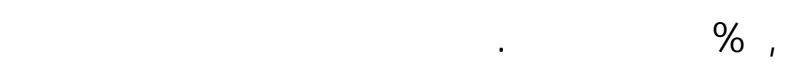
المنخفض في جمبع دول مجلس التعاون الخليجي العربي.

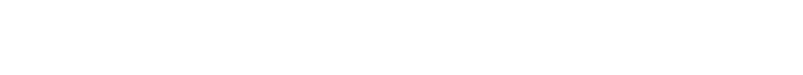

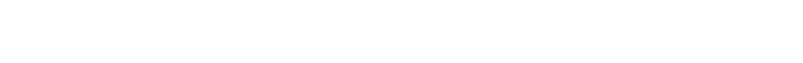

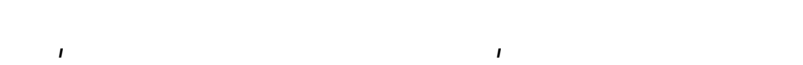
في جنوب الصحراء الكبرى، و, وفي جنوبشرق آلسيا،
مستوى التعال للنخل، حيث التضح أن خط الفقر الطلق

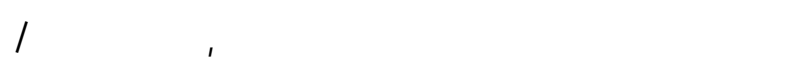
فرد/ للسنة وهوما يعال بr, rV دولار/ تلك الفترة والذي نخله يعالل r rV دولار/ اليوم لم ينخر أي جزء من نخله (الانخار يساوي الصفر) حيث

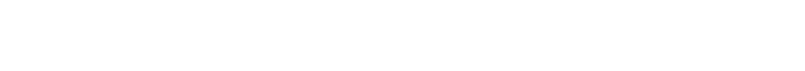
نخله عن هذا الحد لن يسطبع تلبية لحتيلجاته الاستهلاكية.

خلاصة الفرل:

أن غط الفقر الطلق لدول مجلس التعاون الخليجي

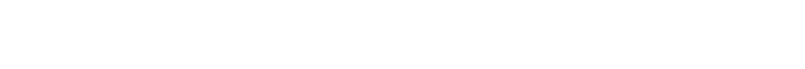

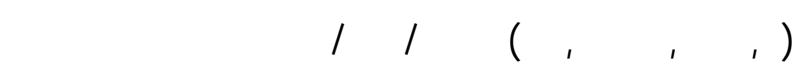
الدول تشتخدم أنمطل لستهلاكية أعلى للرفله في تحديد الفقر

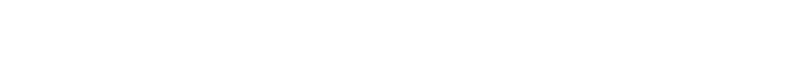

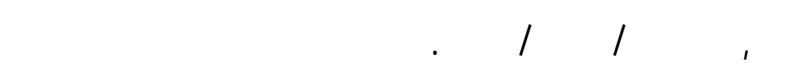

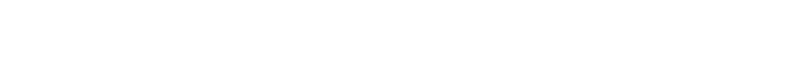
الدول.

ثالثأ: أوضاع قص التغنية بطل مجلس التعاون الخليهي

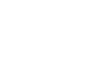

قفيد نقارير مظمة الأغنية والزراعة للأمم المتحة بئن

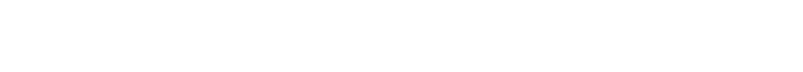

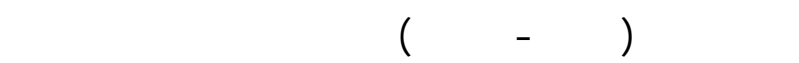

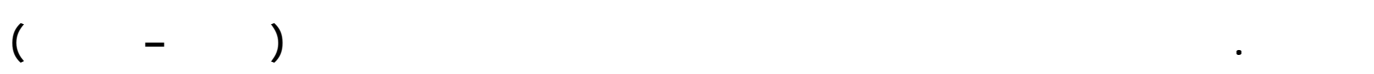

\begin{tabular}{|c|c|c|c|c|c|c|c|c|}
\hline * جو & مؤشر & مسة(أفل) & نهنسة فأ & وزن (\%) & الخلاسبة نالفة & (ص) & نسبة اللسكلن (النغية & \\
\hline$r \cdot \mid \varepsilon$ & 199. & $r \cdot 1 \varepsilon$ & 199. & $\begin{array}{r}-r \cdot{ }_{r} \\
r \cdot{ }^{\prime} \mu\end{array}$ & $\begin{array}{c}-199 . \\
1994\end{array}$ & $\begin{array}{l}-r .11 \\
r .1 \mu\end{array}$ & $199 \%-199$. & \\
\hline$>0$ & $>0$ & - & - & - & - & - & - & الإمارك \\
\hline$>0$ & $V_{1} \cdot \cdot$ & 1,1 & - & $1, V$ & - & 1,0 & rq & الكويت \\
\hline$>0$ & 7,0 & $1, \Lambda$ & $\varepsilon, \mu$ & $0, \mu$ & 11,1 & 1,7 & $\mu$, & للسعوVية \\
\hline$>0$ & $>0$ & 1, & 1,7 & 0,1 & $7, \mu$ & - & - & البحرين \\
\hline$>0$ & $>0$ & $\cdot, 9$ & $\varepsilon, \Lambda$ & $\Lambda, 7$ & $r \mid, \varepsilon$ & - & - & عملن \\
\hline$>0$ & $>0$ & , , & 19 & & & - & & \\
\hline
\end{tabular}

• مؤثر مركب مق تحديد درجاته بلستخدلم ثلاثة معايير هي نسبة اللسكلن ناقصي التغنية، ونسبة السكلن ناقصي الوزن دونسن الخلمسة، ونسبة

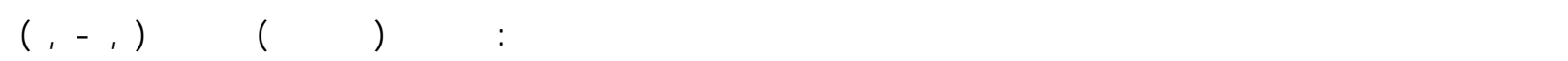

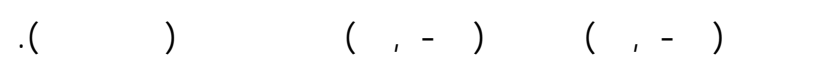

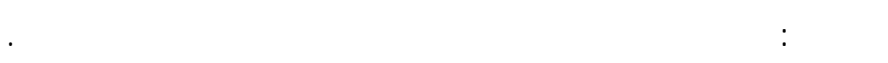


برنلمج الأمم المتحدة الإنمائي، ققرير التمية الب شررية، أع ـداد مختلفة.

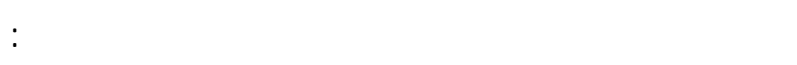
العناصر الرئيسية للاسترالتيجية القضاء على الفقر في البلدان العربية، إدارة الدعم للتنمي ـة والخ ـملت الإداري ـة، الأم م

$$
\text { المتحة، نيويورك. }
$$

تركي بن لهل مي ال ششلاقي 7 . r، الخ صائص الاجتماعي ـة والاقتصاية والديموغرافية لسكلن الأحياء الفقيرة: درلس الفيلة

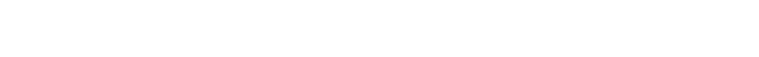
بسالة ملجستير، قم الدرلست الاجتماعية، كلى فة الآداب، جلمعة الملكسعود.

جلمعة الدول العربية، المظمة العربية للتنمية الزراعية سا -10

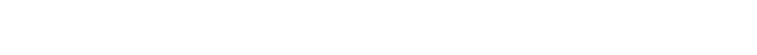
الحدمن الفقر الريفي، صنعاء، اليمن.

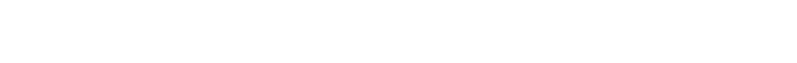

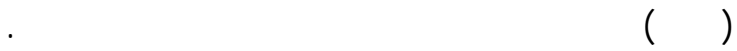
جلمعة الدول العربية، المظمة العربية للتنمية الزراعية، القرير اللسنوي للتنمية الزراعية في الططن العربي، أعداد مختلفة. جلمعة الدول العربية، المظمة العربية للتمية الزراعية، قريب -ر أوضاع الأمن الغذائي العربي، أعداد مختلفة.

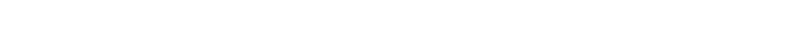
درلسة برلمج ومثروعت الحد من الفقر في المنطق الريفية

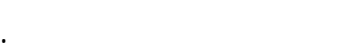
حفصة عبد العطلي علي مبسي 9 ج. r، القيم الكمي الفقر ف ي

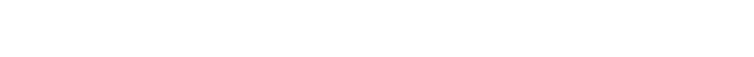
كلية الزراعة، جلمعة عينشهس.

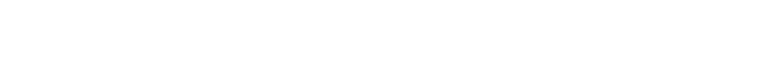
المملكة العربية للسعوية، مجلة العلوم الاجتماعية.

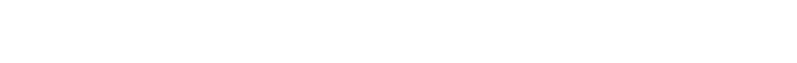

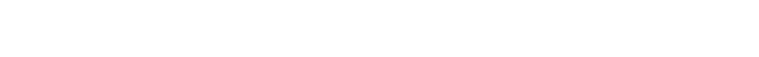
منلسبك رقم (").
وكلن هذا المعل في الثررق الأنف وشمل إفريقيا ؟ب,0

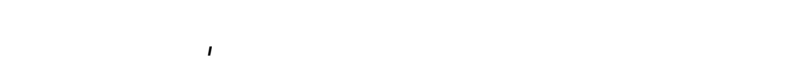

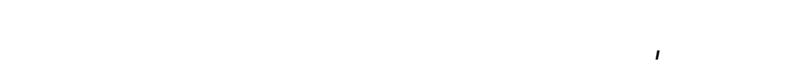
التعاون الخليجي العربي من الدول ذات مؤثرر الجوع المنخفض مقارنة بجمبع دول العالم.

\section{التوصيلت}

\section{في ضوء النتائج التي توطل إليها البهث يمكن التأكيد عل أن.}

دول مجلس التعاون الخليجي العربي ت ستخدم أنم طل ألما

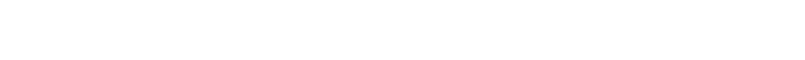
الفقر الهلق العالمي، أي أن خط الفقر لدول مجلس التعاون الخليجي العربي متسق مع الرفاهة الاقتصاية بتلك ال ددول.

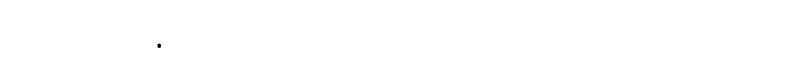

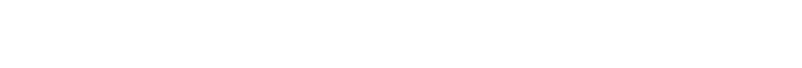
الولايت المتحة الأمريكية وألقل منه في لوك سمبرج ف مي لوني فترتي الدرلسة الأخيرتين.

\section{الم ـرلج ع}

البك الدولي، القرير للسنوي، المجلد الأول، بيانات الدول، أعداد

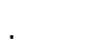

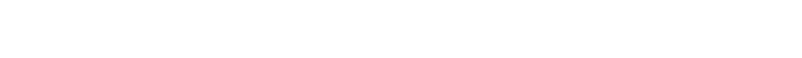
قلم معلثك الضمان الاتماعي في مظقة الرياض، بساض بـالة كتوره، قتم الدرلسك الاجتماعية، جلمعة المالكسعود.

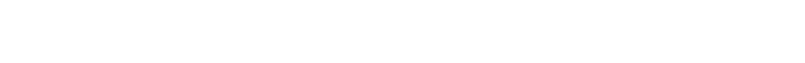

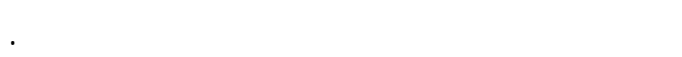

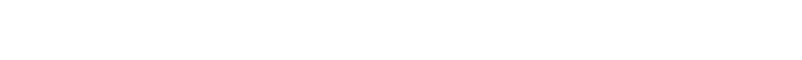
الاقتصادي العربي الموحد، الكويت، أعداد مختلفة.

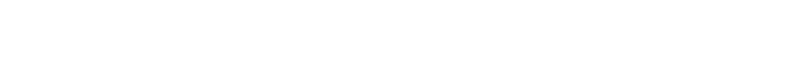

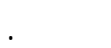




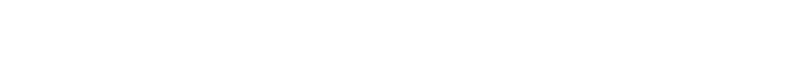

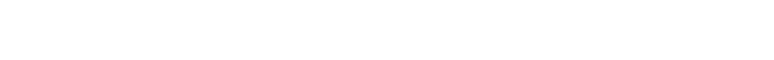

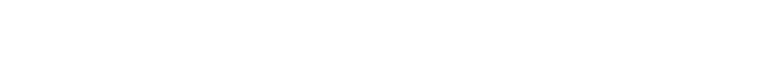
الاجتماعية، كلية الآذاب، جلمعة الملكسعود.

Alderman, H. and Garcia, M. 1993. Poverty, Household Food Security, and Nutrition in Rural Pakistan" International Food Policy Research Institute - Research Report, 96. http://foryoulesson.com/top-10-richest-countries-world.

Bakir M.H .1996. Measuring Poverty in the ESCWA Countries- Economic and Social Commission for Weston Asia Poverty Alleviation Studies relies, No.3- United Nations, New York.

El-Eraky B. Mohamed . 1998. "Income Disparities in Rural Egypt" . Egyptian Journal of Agricultural Economics vol. 8, no. 2, September.

FAO, The state of Food Insecurity in the World, Rome, Different Volumes .IFAD, 2003. Assessment of Rural Poverty: Near East and North Africa, Rome,.

The World Bank. 2002. Poverty reduction and the World Bank, Progress in Operationaling the WDR 2000-2001, Washington D.C.

The World Bank, Poverty Trends and Voices of the Poor: Income Poverty, Washington, Different Volumes.

Chen, Shaohua, and Martin Ravallion. 2012, "More Relatively-Poor People in a Less Absolutely-Poor World," World Bank Policy Research Working Paper 6114 (Washington).
عال عبد الرحمن بوقري 999 ا، مفهوه حد الكفاية في الفك _ر

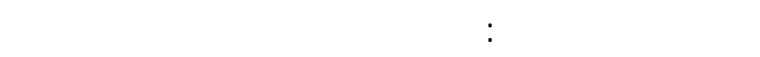

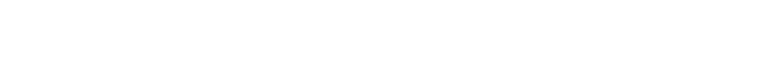

$$
\text { المالك عبد العزيز بجدة . }
$$

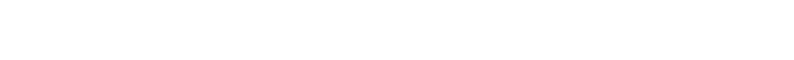

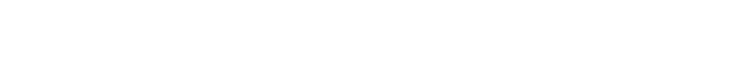

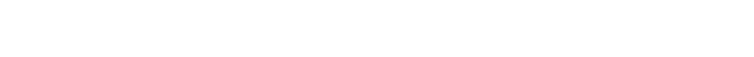

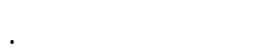

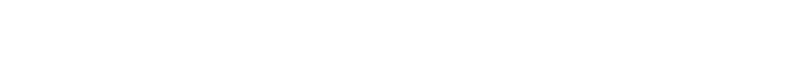

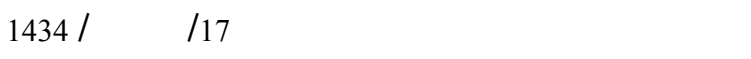

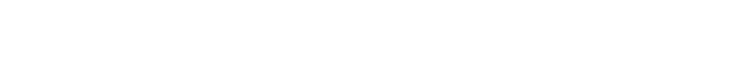
. http://www.mep.gov.sa

مصلحة الإحصاءات العلمة، "بحث الإفاق الاستهلافي للأس ـرة

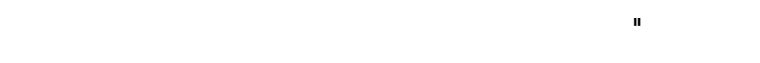
للسعودية.

مظمة الأغذية والزراعة للأمم المتحة، حال ـة النع دلم الأم نـ الغذائي في العالم، روما، أعداد مختلفة. 


\title{
ABSTRACT \\ An Economic Study of the Consistency of the Poverty Line with Welfare in the Arab Gulf Cooperation Council
}

\author{
Maha A. F. Saied
}

The search aims at estimate the absolute poverty line in GCC, for compare it with the international poverty line, to identify the consistent with welfare in those countries. A search depends on World Bank data for the GCC countries during the period (2001-2014) in the cross-series form to estimate consumption and saving functions. To estimate the absolute poverty line at the level of the Arab Gulf Cooperation Council (GCC), dividing the study period (2001-2014) to three periods: the first period (2001-2004), and the second period (2005-2009), and the third period (2010-2014). The results of those periods that increase the per capita income of about \$100/ year, leading to increased consumer spending per capita per year by $47,12,3 \$$ for the three periods, respectively. The absolute poverty line of the individual countries of the Arab Gulf Cooperation Council for the three periods was about 1420.5, 6754.7, 9963.1 USD / capita / year which is equivalent to $3.9,18.5,27.3 \$ /$ person / day for the three periods, respectively. this means that an individual during those periods in which his income equivalent to $3.9,18.5,27.3$ dollars / day has not spared any part of his income (savings is equal to zero), where barely enough to meet consumer needs, and that the individual who is earning less than this limit will not be able meet his needs.

This confirms that those countries use a higher consumption pattern of welfare in determining the poverty, which exceeds global absolute poverty line, which amounts to about $\$ 2.25 /$ person / day. Moreover, the Arab states of the low hunger index compared to all the nations of the world. Therefore, the study suggests that the Gulf Cooperation Council are using the highest consumption patterns of welfare in determining the poverty, which exceeds global absolute poverty line. This means that the GCC Arab poverty line is consistent with the economic welfare of those countries. Hunger Index results also revealed that the GCC Arab states of low hunger index compared to all the nations of the world. In addition, the national absolute poverty line for these countries is higher than in the United States and lower than in Luxembourg in the last two study periods.

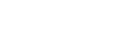

\begin{tabular}{|c|c|c|c|c|c|c|c|}
\hline لإجمالي & الكوبت & قار & عملن & السعولية & البحرين & لإمارك & السنولت \\
\hline$r q, 7$ & $r$ & $\cdot, 7$ & $r, r$ & $r \cdot, 9$ & $\cdot, \mathrm{V}$ & $\mu, 1$ & $r \cdots 1$ \\
\hline$\mu \cdot, \Lambda$ & $r$ & $\cdot, 7$ & $r, \mu$ & $r \mid, \Lambda$ & $\cdot, \mathrm{V}$ & $\mu, r$ & $r \cdots r$ \\
\hline$\mu \Gamma, Y$ & $r, 1$ & $\cdot, \mathrm{V}$ & $r, \varepsilon$ & $r r, q$ & $\cdot, \Lambda$ & $\mu, \varepsilon$ & $r \cdots \mu$ \\
\hline$\mu \mu, V$ & $r, r$ & $\cdot, \mathrm{V}$ & $r, 0$ & $r^{\mu}, \Lambda$ & $\cdot, \Lambda$ & $\mu, V$ & $r \cdots \varepsilon$ \\
\hline$\mu 0, \varepsilon$ & $r, \mu$ & $\cdot, \Lambda$ & $r, 0$ & $r \varepsilon, V$ & $\cdot 9$ & $\varepsilon, 1$ & $r \cdots o$ \\
\hline${ }^{\mu} \mathrm{V}, \mu$ & $r, \varepsilon$ & 1 & $r, 7$ & $r 0, \varepsilon$ & 1 & 0 & $r \cdots 7$ \\
\hline$\mu q, 0$ & $r, 7$ & $1, r$ & $r, 7$ & $r 0,9$ & 1 & $7, r$ & $r \cdots V$ \\
\hline$\varepsilon r, r$ & $r, V$ & $1, \varepsilon$ & $r, \tau$ & $r 7, \varepsilon$ & 1,1 & $\Lambda, 1$ & $r \cdots \Lambda$ \\
\hline$\varepsilon \mu, \mu$ & $r, 9$ & 1,7 & $r, V$ & $r\urcorner, \Lambda$ & $1, r$ & $\Lambda, r$ & $r \ldots q$ \\
\hline$\varepsilon \varepsilon, \mu$ & $\mu$ & $1, V$ & $r, \Lambda$ & $r V, \mu$ & $1, \mu$ & $\Lambda, \mu$ & $r \cdot 1$. \\
\hline$\varepsilon 0, \varepsilon$ & $\mu, 1$ & 1,9 & $\mu$ & $r V, \Lambda$ & $1, \mu$ & $\Lambda, \mu$ & $r .11$ \\
\hline$\varepsilon V, \varepsilon$ & $\mu, \mu$ & $r, 1$ & $\mu, \mu$ & $r \Lambda, \mu$ & $1, \mu$ & $9, r$ & $r \cdot 1 r$ \\
\hline$\varepsilon \Lambda, V$ & $\mu, \varepsilon$ & $r, r$ & $\mu, 7$ & $r \Lambda, \Lambda$ & $1, \mu$ & $9, \mu$ & $r \cdot{ }^{\prime} \mu$ \\
\hline$\varepsilon १, \Lambda$ & $\mu, 0$ & $r, \mu$ & $\mu, q$ & $r q, \varepsilon$ & $1, \mu$ & $9, \varepsilon$ & $r \cdot I \varepsilon$ \\
\hline
\end{tabular}




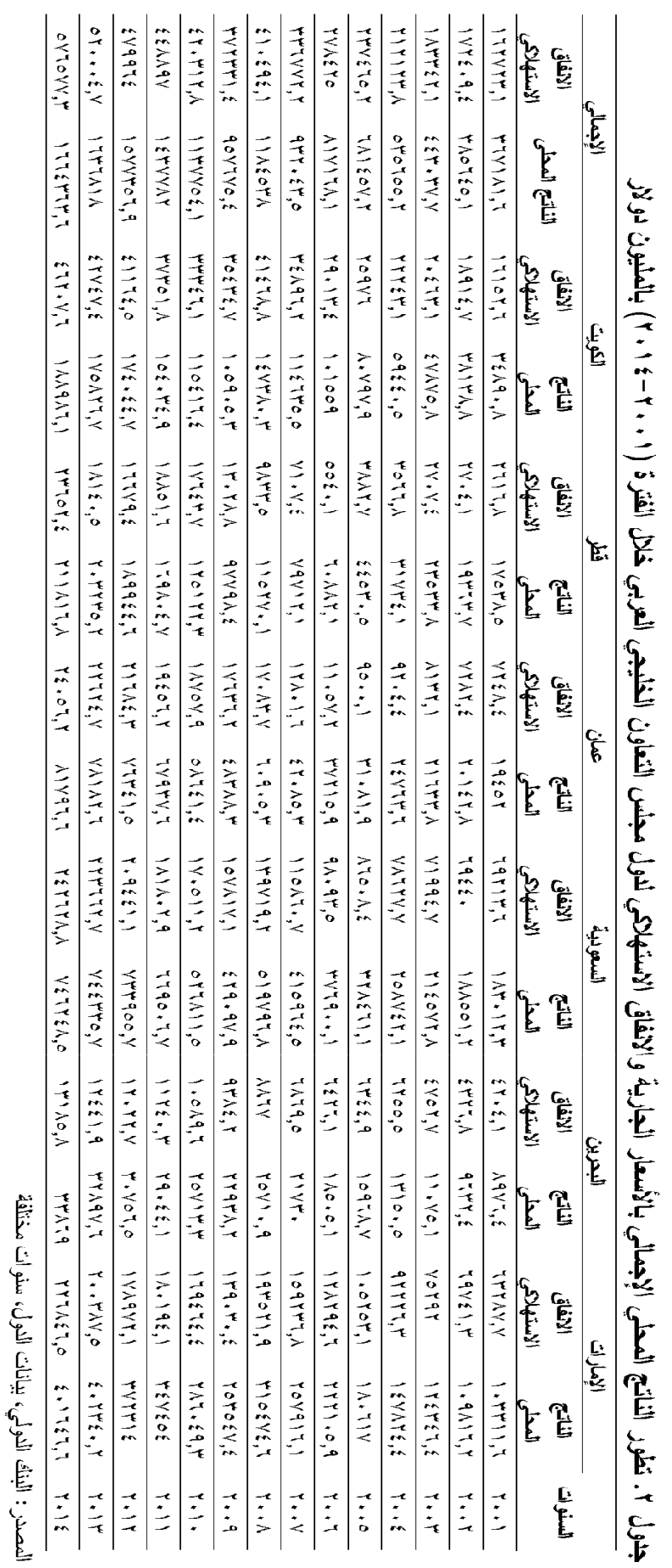

